\title{
FINITE CYCLIC GROUP ACTIONS WITH THE TRACIAL ROKHLIN PROPERTY
}

\author{
N. CHRISTOPHER PHILLIPS
}

\begin{abstract}
We give examples of actions of $\mathbb{Z} / 2 \mathbb{Z}$ on AF algebras and AT algebras which demonstrate the differences between the (strict) Rokhlin property and the tracial Rokhlin property, and between (strict) approximate representability and tracial approximate representability. Specific results include the following. We determine exactly when a product type action of $\mathbb{Z} / 2 \mathbb{Z}$ on a UHF algebra has the tracial Rokhlin property; in particular, unlike for the strict Rokhlin property, every UHF algebra admits such an action. We prove that Blackadar's action of $\mathbb{Z} / 2 \mathbb{Z}$ on the $2^{\infty}$ UHF algebra, whose crossed product is not $\mathrm{AF}$ because it has nontrivial $K_{1}$-group, has the tracial Rokhlin property, and we give an example of an action of $\mathbb{Z} / 2 \mathbb{Z}$ on a simple unital AF algebra which has the tracial Rokhlin property and such that the $K_{0}$-group of the crossed product has torsion. In particular, the crossed product of a simple unital $\mathrm{AF}$ algebra by an action of $\mathbb{Z} / 2 \mathbb{Z}$ with the tracial Rokhlin property need not be AF. We give an example of a strongly selfabsorbing $\mathrm{C}^{*}$-algebra $D$ (the $3^{\infty}$ UHF algebra), a $D$-stable simple separable unital $\mathrm{C}^{*}$-algebra $B$, and an action of $\mathbb{Z} / 2 \mathbb{Z}$ on $B$ with the tracial Rokhlin property such that the crossed product is not $D$-stable. We also give examples of a tracially approximately representable action of $\mathbb{Z} / 2 \mathbb{Z}$ on a simple unital AF algebra which is nontrivial on $K_{0}$, and of a tracially approximately representable action of $\mathbb{Z} / 2 \mathbb{Z}$ on a simple unital AT algebra with real rank zero which is nontrivial on $K_{1}$.
\end{abstract}

\section{INTRODUCTION}

The tracial Rokhlin property for actions of finite groups on $\mathrm{C}^{*}$-algebras was introduced in [27] for the purpose of proving that every simple higher dimensional noncommutative torus is an AT algebra (done in 28]), and proving that certain crossed products of such algebras by finite cyclic groups are AF algebras (done in [6]). The purpose of this paper is to provide other examples of actions of finite cyclic groups with the tracial Rokhlin property on $\mathrm{C}^{*}$-algebras with tracial rank zero. We demonstrate by example the differences between the (strict) Rokhlin property and the tracial Rokhlin property, and between (strict) approximate representability (Definition 3.6(2) of [13]) and its tracial analog, tracial approximate representability (Definition 3.2 of [27]). (To emphasize the distinction with their tracial analogs, in this paper we refer to the strict Rokhlin property and to strict approximate representability.) In [5], Blackadar constructed an action of $\mathbb{Z} / 2 \mathbb{Z}$ on the $2^{\infty}$ UHF algebra such that the crossed product has nontrivial $K_{1}$-group, and is hence not AF. As one of our examples, we prove that this action has the tracial Rokhlin property. Earlier, in one of the exercises (10.11.3) of his book [3], Blackadar

Received by the editors December 30, 2007 and, in revised form, January 29, 2010.

2010 Mathematics Subject Classification. Primary 46L55; Secondary 46L40.

This research was partially supported by NSF grants DMS 0070776 and DMS 0302401. 
gave an example of an order two automorphism of $K_{0}(A)$ for a simple separable AF algebra $A$ such that, if this automorphism could be implemented by an order two automorphism of $A$, then the resulting crossed product by $\mathbb{Z} / 2 \mathbb{Z}$ would have torsion in $K_{0}$ or nontrivial $K_{1}$. With a very slight modification of Blackadar's algebra (we use $\mathbb{Z}\left[\frac{1}{3}\right] \oplus \mathbb{Z}$ instead of $\mathbb{Z}\left[\frac{1}{2}\right] \oplus \mathbb{Z}$ ), our examples include actions of $\mathbb{Z} / 2 \mathbb{Z}$ on this AF algebra with the tracial Rokhlin property such that $K_{0}$ of the crossed product has a summand isomorphic to $\mathbb{Z} / 2^{n} \mathbb{Z}$, and also actions with the tracial Rokhlin property such that $K_{1}$ of the crossed product is nonzero.

Our results give counterexamples to various strengthenings of results in [27] and elsewhere. In particular, using the notation $\mathbb{Z}_{n}$ for $\mathbb{Z} / n \mathbb{Z}$ :

- Even on a UHF algebra, an action of $\mathbb{Z}_{n}$ with the tracial Rokhlin property need not have the strict Rokhlin property, and in fact the crossed product by such an action can have nontrivial $K_{1}$-group, so need not be AF. See Example 3.1. and, for a simple AF algebra which is not UHF, Example 4.5.

- The crossed product of a simple unital AF algebra by an action of $\mathbb{Z}_{n}$ with the tracial Rokhlin property can have torsion in its $K_{0}$-group - even if the action is tracially approximately representable. See Example 4.1.

- If an action $\alpha$ of $\mathbb{Z}_{n}$ on a simple unital $\mathrm{C}^{*}$-algebra $A$ with tracial rank zero is strictly approximately representable and has the tracial Rokhlin property, it does not follow that the automorphisms of the dual action are approximately inner - even if $A$ is UHF and $\alpha$ is locally representable in the sense of Section I of [10. See Example 2.8.

- If an action $\alpha$ of $\mathbb{Z}_{n}$ on a simple unital $\mathrm{C}^{*}$-algebra $A$ with tracial rank zero is tracially approximately representable and has the strict Rokhlin property, it does not follow that the dual action has the strict Rokhlin property - even if $A$ is AT. Use the dual of the action in Example 3.1

- A tracially approximately inner automorphism of a simple unital $\mathrm{C}^{*}$-algebra $A$ with tracial rank zero need not be trivial on $K_{0}(A)$ - even if $A$ is $\mathrm{AF}$ and $\alpha$ generates an action of $\mathbb{Z}_{n}$ with the strict Rokhlin property. Use the dual of the action in Example 2.8.

- A tracially approximately inner automorphism of a simple unital $\mathrm{C}^{*}$-algebra $A$ with tracial rank zero need not be trivial on $K_{1}(A)$ - even if $A$ is AT and $\alpha$ generates an action of $\mathbb{Z}_{n}$ with the strict Rokhlin property. Use the dual of the action in Example 3.1

- There is an action $\alpha$ of $\mathbb{Z}_{n}$ on a simple AF algebra such that $C^{*}\left(\mathbb{Z}_{n}, A, \alpha\right)$ is again a simple AF algebra, but such that $\alpha$ does not have the tracial Rokhlin property. This can happen even when the dual action has the strict Rokhlin property and $\alpha$ is strictly approximately representable, even locally representable in the sense of Section I of [10. See Example 2.9.

- The dual of an action with the tracial Rokhlin property need not have the tracial Rokhlin property, even when both the original algebra and the crossed product are simple AF algebras, and even when the original action in fact has the strict Rokhlin property. Use the dual of the action in Example 2.9.

- If $D$ is a strongly selfabsorbing $\mathrm{C}^{*}$-algebra, $A$ is a simple separable unital $D$-absorbing $\mathrm{C}^{*}$-algebra, and $\alpha: \mathbb{Z}_{n} \rightarrow \operatorname{Aut}(A)$ has the tracial Rokhlin property, it does not follow that the crossed product is $D$-absorbing - even if $A$ and $C^{*}\left(\mathbb{Z}_{n}, A, \alpha\right)$ have tracial rank zero. See Example 4.11. 
Examples involving actions on simple $\mathrm{C}^{*}$-algebras which do not have tracial rank zero, many of them on simple $\mathrm{C}^{*}$-algebras with tracial rank one, will appear in 23.

The first section contains preliminaries. In particular, we recall the most important definitions, and we give a new criterion for an action to have the tracial Rokhlin property. The second section contains three examples of product type actions of $\mathbb{Z}_{2}$ on the $2^{\infty}$ UHF algebra. Along the way, we give a criterion for exactly when such a product type action has the tracial Rokhlin property. The third section contains the proof that Blackadar's action has the tracial Rokhlin property. The fourth section gives the examples of the kind suggested by 10.11 .3 of [3]. In the last section, we state some open problems.

This paper contains the results in Section 12 of the unpublished preprint [26], with some of the examples put in a more general framework, as well as some of the material from Section 13. Section 4 here is entirely new.

We use the notation $\mathbb{Z}_{n}$ for $\mathbb{Z} / n \mathbb{Z}$; the $p$-adic integers will not appear in this paper. If $A$ is a $\mathrm{C}^{*}$-algebra and $\alpha: A \rightarrow A$ is an automorphism such that $\alpha^{n}=$ $\operatorname{id}_{A}$, then we write $C^{*}\left(\mathbb{Z}_{n}, A, \alpha\right)$ for the crossed product of $A$ by the action of $\mathbb{Z}_{n}$ generated by $\alpha$. We write $A_{\text {sa }}$ for the set of selfadjoint elements of a $\mathrm{C}^{*}$-algebra $A$. We write $p \precsim q$ to mean that the projection $p$ is Murray-von Neumann equivalent to a subprojection of $q$, and $p \sim q$ to mean that $p$ is Murray-von Neumann equivalent to $q$. Also, $[a, b]$ denotes the additive commutator $a b-b a$. We denote by $T(A)$ the set of all tracial states on a unital $\mathrm{C}^{*}$-algebra $A$, equipped with the weak* topology. For any element of $T(A)$, we use the same letter for its standard extension to $M_{n}(A)$ for arbitrary $n$, and to $M_{\infty}(A)=\bigcup_{n=1}^{\infty} M_{n}(A)$ (no closure). We take $\mathbb{N}=\{n \in \mathbb{Z}: n>0\}$.

\section{The tracial RokHLin PROPERTY \\ AND TRACIAL APPROXIMATE REPRESENTABILITY}

We recall the properties we will use. Since we will be almost exclusively concerned with stably finite $\mathrm{C}^{*}$-algebras, we give the simpler versions of the tracial Rokhlin property and of tracial approximate representability that are valid for the finite case. The following is Definition 1.1 of [27]. It is essentially Definition 3.1 of [13, although that definition is stated in terms of central sequences. See the discussion after Definition 1.1 of [27] for the equivalence of the two definitions. We furthermore call the condition the strict Rokhlin property, to emphasize the distinction with the tracial Rokhlin property.

Definition 1.1. Let $A$ be a unital $\mathrm{C}^{*}$-algebra, and let $\alpha: G \rightarrow \operatorname{Aut}(A)$ be an action of a finite group $G$ on $A$. We say that $\alpha$ has the strict Rokhlin property if for every finite set $F \subset A$ and every $\varepsilon>0$, there are mutually orthogonal projections $e_{g} \in A$ for $g \in G$ such that:

(1) $\left\|\alpha_{g}\left(e_{h}\right)-e_{g h}\right\|<\varepsilon$ for all $g, h \in G$.

(2) $\left\|e_{g} a-a e_{g}\right\|<\varepsilon$ for all $g \in G$ and all $a \in F$.

(3) $\sum_{g \in G} e_{g}=1$.

The following is a special case of Lemma 1.16 of [27. We specialize to cyclic groups because we only use cyclic groups in this paper, and to establish notation for the projections used in the tracial Rokhlin property for finite cyclic groups. 
Lemma 1.2. Let $A$ be a finite infinite dimensional simple unital $C^{*}$-algebra, and let $\alpha \in \operatorname{Aut}(A)$ satisfy $\alpha^{n}=\mathrm{id}_{A}$. The action of $\mathbb{Z}_{n}$ generated by $\alpha$ has the tracial Rokhlin property if and only if for every finite set $F \subset A$, every $\varepsilon>0$, and every nonzero positive element $x \in A$, there are mutually orthogonal projections $e_{0}, e_{1}, \ldots, e_{n-1} \in A$ such that:

(1) $\left\|\alpha\left(e_{j}\right)-e_{j+1}\right\|<\varepsilon$ for $0 \leq j \leq n-1$, where by convention we take the indices $\bmod n$, that is, $e_{n}=e_{0}$.

(2) $\left\|e_{j} a-a e_{j}\right\|<\varepsilon$ for $0 \leq j \leq n-1$ and all $a \in F$.

(3) With $e=\sum_{j=0}^{n-1} e_{j}$, the projection $1-e$ is Murray-von Neumann equivalent to a projection in the hereditary subalgebra of $A$ generated by $x$.

The following definition is equivalent to Definition 3.6(2) of 13 . The definition of 13 is actually stated in terms of central sequences, but it is equivalent to our definition by Lemma 3.1 of [27. Also, we use the term strictly approximately representable to emphasize the distinction with tracially approximately representable.

Definition 1.3. Let $A$ be a unital $\mathrm{C}^{*}$-algebra. An action $\alpha: G \rightarrow \operatorname{Aut}(A)$ of a finite abelian group $G$ on $A$ is strictly approximately representable if for every finite set $F \subset A$ and every $\varepsilon>0$, there are unitaries $w_{g} \in A$ for $g \in G$ such that:

(1) $\left\|\alpha_{g}(a)-w_{g} a w_{g}^{*}\right\|<\varepsilon$ for all $a \in F$ and all $g \in G$.

(2) $\left\|w_{g} w_{h}-w_{g h}\right\|<\varepsilon$ for all $g, h \in G$.

(3) $\left\|\alpha_{g}\left(w_{h}\right)-w_{h}\right\|<\varepsilon$ for all $g, h \in G$.

Lemma 1.4 (Lemma 3.4 of [27]). Let $A$ be a finite infinite dimensional simple unital $C^{*}$-algebra, and let $\alpha: G \rightarrow \operatorname{Aut}(A)$ be an action of a finite abelian group $G$ on $A$. Then $\alpha$ is tracially approximately representable if and only if for every finite set $F \subset A$, every $\varepsilon>0$, and every positive element $x \in A$ with $\|x\|=1$, there are a projection $e \in A$ and unitaries $w_{g} \in$ eAe for $g \in G$ such that:

(1) $\|e a-a e\|<\varepsilon$ for all $a \in F$.

(2) $\| \alpha_{g}($ eae $)-w_{g}$ eaew $w_{g}^{*} \|<\varepsilon$ for all $a \in F$ and all $g \in G$.

(3) $\left\|w_{g} w_{h}-w_{g h}\right\|<\varepsilon$ for all $g, h \in G$.

(4) $\left\|\alpha_{g}\left(w_{h}\right)-w_{h}\right\|<\varepsilon$ for all $g, h \in G$.

(5) $1-e$ is Murray-von Neumann equivalent to a projection in the hereditary subalgebra of $A$ generated by $x$.

We have the following duality between the (tracial) Rokhlin property and (tracial) approximate representability.

Theorem 1.5 (Lemma 3.8 of 13 ). Let $A$ be a unital $C^{*}$-algebra, and let $\alpha: G \rightarrow$ $\operatorname{Aut}(A)$ be an action of a finite abelian group $G$ on $A$. Then:

(1) $\alpha$ has the strict Rokhlin property if and only if $\widehat{\alpha}$ is strictly approximately representable.

(2) $\alpha$ is strictly approximately representable if and only if $\widehat{\alpha}$ has the strict Rokhlin property. 
Theorem 1.6 (Theorem 3.11 of [27]). Let $A$ be an infinite dimensional simple unital $C^{*}$-algebra, and let $\alpha: G \rightarrow \operatorname{Aut}(A)$ be an action of a finite abelian group $G$ on $A$ such that $C^{*}(G, A, \alpha)$ is also simple. Then:

(1) $\alpha$ has the tracial Rokhlin property if and only if $\widehat{\alpha}$ is tracially approximately representable.

(2) $\alpha$ is tracially approximately representable if and only if $\widehat{\alpha}$ has the tracial Rokhlin property.

Remark 1.7. We point out that if $A$ is an infinite dimensional simple unital $\mathrm{C}^{*}$ algebra, and if $\alpha: G \rightarrow \operatorname{Aut}(A)$ is a tracially approximately representable action of a finite abelian group $G$ on $A$, then each $\alpha_{g}$ is tracially approximately inner in the sense of Definition 5.1 of [27].

We now give a useful criterion for an action to have the tracial Rokhlin property.

Lemma 1.8. Let $A$ be a separable infinite dimensional simple unital $C^{*}$-algebra with tracial rank zero. Let $\alpha: G \rightarrow \operatorname{Aut}(A)$ be an action of a finite group $G$ on $A$. Suppose that for every finite set $F \subset A$ and every $\varepsilon>0$ there are positive elements $a_{g} \in A$ for $g \in G$, with $0 \leq a_{g} \leq 1$, such that:

(1) $a_{g} a_{h}=0$ for $g, h \in G$ with $g \neq h$.

(2) $\left\|\alpha_{g}\left(a_{h}\right)-a_{g h}\right\|<\varepsilon$ for all $g, h \in G$.

(3) $\left\|a_{g} c-c a_{g}\right\|<\varepsilon$ for all $g \in G$ and $c \in F$.

(4) $\tau\left(1-\sum_{g \in G} a_{g}\right)<\varepsilon$ for every tracial state $\tau$ on $A$.

Then $\alpha$ has the tracial Rokhlin property.

Proof. We verify the criterion of Theorem 5.3 of [6] using conditions (1), (2), and $\left(3^{\prime}\right)$ there. Thus, let $S \subset A$ be finite and let $\varepsilon>0$. Set $n=\operatorname{card}(G)$. Choose $\varepsilon_{0}>0$ so small that $2 \sqrt{(2 n+1) \varepsilon_{0}}+(2 n+5) \varepsilon_{0} \leq \varepsilon$.

Apply the hypothesis with $S$ as given and with $\varepsilon_{0}$ in place of $\varepsilon$. For each $g \in G$, use real rank zero to choose a projection $e_{g} \in \overline{a_{g} A a_{g}}$ such that $\left\|e_{g} a_{g}-a_{g}\right\|<\varepsilon_{0}$ and $\left\|a_{g} e_{g}-a_{g}\right\|<\varepsilon_{0}$. Then $\left\|e_{g} a_{g} e_{g}-a_{g}\right\|<2 \varepsilon_{0}$. So

$$
\sum_{h \in G} \tau\left(e_{h} a_{h} e_{h}\right)>\sum_{h \in G} \tau\left(a_{h}\right)-2 n \varepsilon_{0}>1-(2 n+1) \varepsilon_{0} .
$$

Since $\sum_{h \in G} \tau\left(e_{h}\right) \leq 1$ (because the $e_{h}$ are orthogonal) and $e_{h} a_{h} e_{h} \leq e_{h}$ for all $h \in G$, we get, for each $g \in G$ and $\tau \in T(A)$,

$$
\tau\left(e_{g}\right) \leq 1-\sum_{h \neq g} \tau\left(e_{h}\right) \leq 1-\sum_{h \neq g} \tau\left(e_{h} a_{h} e_{h}\right)<\tau\left(e_{g} a_{g} e_{g}\right)+(2 n+1) \varepsilon_{0} .
$$

Therefore $0 \leq \tau\left(e_{g}-e_{g} a_{g} e_{g}\right)<(2 n+1) \varepsilon_{0}$.

Recall the $L^{2}$-norm (or seminorm) associated with a tracial state $\tau$ of a $\mathrm{C}^{*}$ algebra $A$, given by $\|a\|_{2, \tau}=\tau\left(a^{*} a\right)^{1 / 2}$, as used in Section 5 of [6]. Using $\left\|\left(e_{g}-e_{g} a_{g} e_{g}\right)^{1 / 2}\right\| \leq 1$ at the third step, we now have

$$
\begin{aligned}
\left\|e_{g}-e_{g} a_{g} e_{g}\right\|_{2, \tau} & \leq\left\|\left(e_{g}-e_{g} a_{g} e_{g}\right)^{1 / 2}\right\|_{2, \tau}\left\|\left(e_{g}-e_{g} a_{g} e_{g}\right)^{1 / 2}\right\| \\
& \leq \tau\left(e_{g}-e_{g} a_{g} e_{g}\right)^{1 / 2}<\sqrt{(2 n+1) \varepsilon_{0}},
\end{aligned}
$$

so that

$$
\left\|e_{g}-a_{g}\right\|_{2, \tau} \leq\left\|e_{g}-e_{g} a_{g} e_{g}\right\|_{2, \tau}+\left\|e_{g} a_{g} e_{g}-a_{g}\right\|<\sqrt{(2 n+1) \varepsilon_{0}}+2 \varepsilon_{0} .
$$


Now we can verify conditions (1), (2), and $\left(3^{\prime}\right)$ of Theorem 5.3 of [6]. Let $\tau \in T(A)$. Then for $g, h \in G$ we have

$$
\begin{aligned}
\left\|\alpha_{g}\left(e_{h}\right)-e_{g h}\right\|_{2, \tau} & \leq\left\|e_{h}-a_{h}\right\|_{2, \tau}+\left\|e_{g h}-a_{g h}\right\|_{2, \tau}+\left\|\alpha_{g}\left(a_{h}\right)-a_{g h}\right\| \\
& <2\left(\sqrt{(2 n+1) \varepsilon_{0}}+2 \varepsilon_{0}\right)+\varepsilon_{0} \leq \varepsilon,
\end{aligned}
$$

and for $g \in G$ and $c \in S$ we have

$$
\left\|\left[e_{g}, c\right]\right\|_{2, \tau} \leq 2\left\|e_{g}-a_{g}\right\|_{2, \tau}+\left\|\left[a_{g}, c\right]\right\|<2\left(\sqrt{(2 n+1) \varepsilon_{0}}+2 \varepsilon_{0}\right)+\varepsilon_{0} \leq \varepsilon .
$$

Also, using

$$
\tau\left(e_{g}\right) \geq \tau\left(e_{g} a_{g} e_{g}\right) \geq \tau\left(a_{g}\right)-\left\|a_{g}-e_{g} a_{g} e_{g}\right\|>\tau\left(a_{g}\right)-2 \varepsilon_{0},
$$

we get

$$
\tau\left(1-\sum_{g \in G} e_{g}\right)<(2 n+1) \varepsilon_{0} \leq \varepsilon .
$$

An application of Theorem 5.3 of [6] completes the proof.

Theorem 1.9. Let $A$ be a separable infinite dimensional simple unital $C^{*}$-algebra with tracial rank zero. Let $\alpha: G \rightarrow \operatorname{Aut}(A)$ be an action of a finite group $G$ on $A$. Suppose that for every finite set $F \subset A$ and every $\varepsilon>0$ there are positive elements $a_{g} \in A$ for $g \in G$, with $0 \leq a_{g} \leq 1$, such that:

(1) $\left\|a_{g} a_{h}\right\|<\varepsilon$ for $g, h \in G$ with $g \neq h$.

(2) $\left\|\alpha_{g}\left(a_{h}\right)-a_{g h}\right\|<\varepsilon$ for all $g, h \in G$.

(3) $\left\|a_{g} c-c a_{g}\right\|<\varepsilon$ for all $g \in G$ and $c \in F$.

(4) $\tau\left(1-\sum_{g \in G} a_{g}\right)<\varepsilon$ for every tracial state $\tau$ on $A$.

Then $\alpha$ has the tracial Rokhlin property.

Proof. We prove that the hypotheses imply those of Lemma 1.8 Let $F \subset A$ be finite and let $\varepsilon>0$. Without loss of generality $\|c\| \leq 1$ for all $c \in F$. Let $B$ be the universal (nonunital) $\mathrm{C}^{*}$-algebra generated by selfadjoint elements $d_{g}$ for $g \in G$, subject to the relations $0 \leq d_{g} \leq 1$ and $d_{g} d_{h}=0$ for $g \neq h$. Let $n=\operatorname{card}(G)$. Then $B$ is isomorphic to the cone over $\mathbb{C}^{n}$, and is hence a projective $C^{*}$-algebra. (See Lemmas 8.1.3 and 10.1.5 and Theorem 10.1.11 of [20].) Therefore it is semiprojective (Definition 14.1.3 of [20]). Thus, using Theorem 14.1.4 of [20] (see Definition 14.1.1 of [20], and take $B$ there to be $\{0\}$ ), there is $\delta>0$ such that whenever $D$ is a $\mathrm{C}^{*}$-algebra and $d_{g} \in D$, for $g \in G$, are positive elements with $0 \leq d_{g} \leq 1$ and such that $\left\|d_{g} d_{h}\right\|<\delta$ for $g \neq h$, then there are positive elements $a_{g} \in D$ for $g \in G$ with $0 \leq a_{g} \leq 1$ such that $a_{g} a_{h}=0$ for $g \neq h$ and

$$
\left\|a_{g}-d_{g}\right\|<\min \left(\frac{1}{4} \varepsilon, \frac{1}{2} n^{-1} \varepsilon\right)
$$

for all $g \in G$. Apply our hypotheses with $F$ as given and with $\min \left(\frac{1}{2} \varepsilon, \delta\right)$ in place of $\varepsilon$, obtaining $d_{g} \in A$ for $g \in G$, and let $a_{g} \in A$ be as above. The relation $a_{g} a_{h}=0$ for $g \neq h$ is condition (11) of Lemma 1.8, For (2), estimate

$$
\left\|\alpha_{g}\left(a_{h}\right)-a_{g h}\right\| \leq\left\|\alpha_{g}\left(d_{h}\right)-d_{g h}\right\|+\left\|a_{h}-d_{h}\right\|+\left\|a_{g h}-d_{g h}\right\|<\frac{1}{2} \varepsilon+\frac{1}{4} \varepsilon+\frac{1}{4} \varepsilon=\varepsilon .
$$

For (3), use $\|c\| \leq 1$ for $c \in F$ to estimate

$$
\left\|a_{g} c-c a_{g}\right\| \leq\left\|d_{g} c-c d_{g}\right\|+2\left\|a_{g}-d_{g}\right\|<\frac{1}{2} \varepsilon+2\left(\frac{1}{4} \varepsilon\right)=\varepsilon .
$$

Condition (4) follows from $\left\|a_{g}-d_{g}\right\|<\frac{1}{2} n^{-1} \varepsilon$ for $g \in G$ and $\tau\left(1-\sum_{g \in G} d_{g}\right)<\frac{1}{2} \varepsilon$. This completes the proof. 


\section{Product type actions}

The three main examples in this section are product type actions of $\mathbb{Z}_{2}$ on the $2^{\infty}$ UHF algebra $D$. In each case we represent $D$ as an infinite tensor product, and the automorphism generating the action as an infinite tensor product of inner automorphisms.

It is useful to first develop some theory. For simplicity, we consider only actions of $\mathbb{Z}_{2}$. This class of actions has been considered several times before, particularly in [8] and [16] (which concentrate on the fixed point algebras) and in [10]. For the convenience of the reader, and to establish notation, we start from scratch. There is, however, some overlap with earlier work. The formula in Lemma 2.3(5) is given, in different terms, in 1.3 of [8]. Lemma 1.6.1 of [8] gives the equivalence of (11) and (3) of Proposition 2.4, together with several other equivalent conditions similar to (1). Example III.4 of [10] contains the equivalence of (3) and (4) of Proposition 2.5, and the final statement. The equivalence of (2) and (3) in Proposition2.6 is Lemma 1.4.1 of 8 .

A complete description of the ordered K-theory of the crossed product seems complicated, except when it is a UHF algebra. We say little about it here. A partial description can be found in 2.2 of [8]. (It is actually intended for the fixed point algebra, but the situation considered also covers crossed products.) A different partial description can be found in Example III.4 of [10].

Lemma 2.1. Let $D$ be an infinite tensor product $C^{*}$-algebra and let $\alpha \in \operatorname{Aut}(D)$ be an automorphism of order two, of the form

$$
D=\bigotimes_{n=1}^{\infty} M_{k(n)} \quad \text { and } \quad \alpha=\bigotimes_{n=1}^{\infty} \operatorname{Ad}\left(p_{n}-q_{n}\right),
$$

with $k(n) \in \mathbb{N}$ and where $p_{n}, q_{n} \in M_{k(n)}$ are projections with $p_{n}+q_{n}=1$. Let $D_{n}=\bigotimes_{m=1}^{n} M_{k(m)}$, so that $D=\lim _{\longrightarrow} D_{n}$, and write $D_{n}=M_{t(n)}$, where $t(n)=$ $\prod_{m=1}^{n} k(m)$. Then the direct system of crossed products can be identified as

$$
C^{*}\left(\mathbb{Z}_{2}, D_{n}\right) \cong M_{t(n)} \oplus M_{t(n)},
$$

with the dual action given by the flip $\sigma_{n}(a, b)=(b, a)$ for $a, b \in M_{t(n)}$, and where the maps

of the direct system are given by

$$
\psi_{n}: M_{t(n-1)} \oplus M_{t(n-1)} \rightarrow M_{t(n)} \oplus M_{t(n)}
$$

$$
\psi_{n}(a, b)=\left(a \otimes p_{n}+b \otimes q_{n}, b \otimes p_{n}+a \otimes q_{n}\right)
$$

for $a, b \in M_{t(n-1)}$. Moreover:

(1) $C^{*}\left(\mathbb{Z}_{2}, D, \alpha\right)$ is a unital AF algebra.

(2) $K_{0}\left(C^{*}\left(\mathbb{Z}_{2}, D, \alpha\right)\right)$ is the direct limit of the system

$$
\mathbb{Z}^{2} \stackrel{T_{1}}{\longrightarrow} \mathbb{Z}^{2} \stackrel{T_{2}}{\longrightarrow} \mathbb{Z}^{2} \stackrel{T_{3}}{\longrightarrow} \cdots,
$$

in which the $n$th term is identified with $K_{0}\left(M_{t(n)} \oplus M_{t(n)}\right)$ in the obvious way, with

$$
T_{n}=\left(\begin{array}{cc}
\operatorname{rank}\left(p_{n}\right) & \operatorname{rank}\left(q_{n}\right) \\
\operatorname{rank}\left(q_{n}\right) & \operatorname{rank}\left(p_{n}\right)
\end{array}\right) .
$$

The isomorphism identifies $\widehat{\alpha}_{*}$ with the direct limit of the maps $(j, k) \mapsto$ $(k, j)$ on $\mathbb{Z}^{2}$. 
(3) The action of $\mathbb{Z}_{2}$ generated by $\alpha$ is strictly approximately representable (Definition 1.31).

(4) The dual action on $C^{*}\left(\mathbb{Z}_{2}, D, \alpha\right)$ has the strict Rokhlin property (Definition 1.1).

(5) For any subset $S \subset \mathbb{N}$, the automorphism $\alpha$ is unchanged if $p_{n}$ and $q_{n}$ are exchanged for every $n \in S$.

Proof. For $n \in \mathbb{N}$ and a unitary $v \in M_{n}$ with $v^{2}=1$, we use the isomorphism $C^{*}\left(\mathbb{Z}_{2}, M_{n}, \operatorname{Ad}(v)\right) \rightarrow M_{n} \oplus M_{n}$ which sends $a \in M_{n}$ to $(a, a)$ and the canonical unitary of the crossed product to $(v,-v)$. The identification of the direct system of crossed products is then a calculation, which we omit. The K-theory computation in part (2) follows. Parts (1) and (3) are immediate. Part (4) follows from part (3) and Theorem 1.5(2). Part (5) follows from the fact that $\operatorname{Ad}(-w)=\operatorname{Ad}(w)$.

Notation 2.2. For a product type automorphism $\alpha$ as in Lemma 2.1 we assume, as Lemma 2.15) says we may, that $\operatorname{rank}\left(p_{n}\right) \geq \operatorname{rank}\left(q_{n}\right)$ for all $n$. We then define

$$
\lambda_{n}=\frac{\operatorname{rank}\left(p_{n}\right)-\operatorname{rank}\left(q_{n}\right)}{\operatorname{rank}\left(p_{n}\right)+\operatorname{rank}\left(q_{n}\right)} \geq 0
$$

for $n \in \mathbb{N}$, and, for $m \leq n$,

$$
\Lambda(m, n)=\lambda_{m+1} \lambda_{m+2} \cdots \lambda_{n} \text { and } \Lambda(m, \infty)=\lim _{n \rightarrow \infty} \Lambda(m, n) .
$$

We furthermore use $\alpha$ interchangeably for the action of $\mathbb{Z}_{2}$ and for the nontrivial automorphism which generates it, and we use $\widehat{\alpha}$ interchangeably for the dual action of $\mathbb{Z}_{2}$ on $C^{*}\left(\mathbb{Z}_{2}, D, \alpha\right)$ and for the nontrivial automorphism which generates it.

It is useful to be able to condense the infinite tensor product description by omitting intermediate terms.

Lemma 2.3. Let $D$ and $\alpha \in \operatorname{Aut}(D)$ be of the form

$$
D=\bigotimes_{n=1}^{N} M_{k(n)} \quad \text { and } \quad \alpha=\bigotimes_{n=1}^{N} \operatorname{Ad}\left(p_{n}-q_{n}\right),
$$

with $k(n) \in \mathbb{N}$ and where $p_{n}, q_{n} \in M_{k(n)}$ are projections with $p_{n}+q_{n}=1$ and $\operatorname{rank}\left(p_{n}\right) \geq \operatorname{rank}\left(q_{n}\right)$. Set $t=\prod_{n=1}^{N} k(n)$. Then there exist projections $p, q \in M_{t}$, with $p+q=1$ and $\operatorname{rank}(p) \geq \operatorname{rank}(q)$, such that there is an isomorphism $D \cong M_{t}$ which intertwines $\alpha$ with $\operatorname{Ad}(p-q)$. Moreover:

(1) With $T_{n}$ as in Lemma 2.1(2), the ranks of $p$ and $q$ are determined by the equation

$$
\left(\begin{array}{cc}
\operatorname{rank}(p) & \operatorname{rank}(q) \\
\operatorname{rank}(q) & \operatorname{rank}(p)
\end{array}\right)=T_{N} T_{N-1} \cdots T_{1}
$$

(2) If for some $n$ we have $\operatorname{rank}\left(p_{n}\right)=\operatorname{rank}\left(q_{n}\right)$, then $\operatorname{rank}(p)=\operatorname{rank}(q)$.

(3) If for all $n$ we have $\operatorname{rank}\left(p_{n}\right)>\operatorname{rank}\left(q_{n}\right)$, then $\operatorname{rank}(p)>\operatorname{rank}(q)$.

(4) If for some $n$ we have $\operatorname{rank}\left(q_{n}\right)>0$, then $\operatorname{rank}(q)>0$.

(5) We have

$$
\frac{\operatorname{rank}(p)-\operatorname{rank}(q)}{\operatorname{rank}(p)+\operatorname{rank}(q)}=\prod_{n=1}^{N} \frac{\operatorname{rank}\left(p_{n}\right)-\operatorname{rank}\left(q_{n}\right)}{\operatorname{rank}\left(p_{n}\right)+\operatorname{rank}\left(q_{n}\right)} .
$$


Proof. For all parts, the general case follows from the case $N=2$ by induction, so we do only that case. Set

$$
p=p_{1} \otimes p_{2}+q_{1} \otimes q_{2} \quad \text { and } \quad q=p_{1} \otimes q_{2}+q_{1} \otimes p_{2} .
$$

Then one checks that $p+q=1$ and $\left(p_{1}-q_{1}\right) \otimes\left(p_{2}-q_{2}\right)=p-q$. Taking the differences of the dimensions of the eigenspaces, we get

$$
\operatorname{rank}(p)-\operatorname{rank}(q)=\left[\operatorname{rank}\left(p_{1}\right)-\operatorname{rank}\left(q_{1}\right)\right]\left[\operatorname{rank}\left(p_{2}\right)-\operatorname{rank}\left(q_{2}\right)\right] .
$$

So $\operatorname{rank}(p) \geq \operatorname{rank}(q)$. We have now proved the main statement and part (1). Parts (2), (3), and (4) are immediate. Part (5) follows by dividing the last equation by $t=k(1) k(2)$.

The implication from (3) to (1) in the following lemma is related to Example 3.2 of 13 .

Proposition 2.4. Let $\alpha \in \operatorname{Aut}(D)$ be a product type automorphism of order 2 as in Lemma 2.1, and adopt Notation 2.2. Then the following are equivalent:

(1) The action $\alpha$ has the strict Rokhlin property.

(2) The dual action $\widehat{\alpha}$ is strictly approximately representable.

(3) There are infinitely many $n \in \mathbb{N}$ such that $\operatorname{rank}\left(p_{n}\right)=\operatorname{rank}\left(q_{n}\right)$ (that is, $\left.\lambda_{n}=0\right)$.

(4) $C^{*}\left(\mathbb{Z}_{2}, D, \alpha\right)$ is a UHF algebra.

(5) $K_{0}\left(C^{*}\left(\mathbb{Z}_{2}, D, \alpha\right)\right)$ is totally ordered.

(6) $\widehat{\alpha}_{*}$ is trivial on $K_{0}\left(C^{*}\left(\mathbb{Z}_{2}, D, \alpha\right)\right)$.

Proof. That (11) implies (2) follows from Theorem 1.5(1). It is immediate that (4) implies (5). Also, (2) implies (6) because approximately inner automorphisms are trivial on K-theory.

We prove that (3) implies (1) and (4). Using Lemma 2.3(2), we may pass to a subsystem and assume that $\operatorname{rank}\left(p_{n}\right)=\operatorname{rank}\left(q_{n}\right)$ for all $n$. Then for every $n$ there is $v_{n} \in M_{k(n)}$ such that $v_{n} v_{n}^{*}=p_{n}$ and $v_{n}^{*} v_{n}=q_{n}$.

To verify the strict Rokhlin property, thus proving (11), it suffices to consider finite subsets $F$ of the dense subalgebra $\bigcup_{n=1}^{\infty} D_{n} \subset D$. Then there is $N$ such that $F \subset D_{N}$. Define $f \in M_{k(N+1)}$ by $f=\frac{1}{2}\left(p_{N+1}+q_{N+1}+v_{N+1}+v_{N+1}^{*}\right)$. Computations show that $f$ is a projection and $\left(p_{N+1}-q_{N+1}\right) f\left(p_{N+1}-q_{N+1}\right)=1-f$. The projections required for the strict Rokhlin property using the given set $F$ and any $\varepsilon>0$ are then

$$
e_{0}=1_{D_{N}} \otimes f, e_{1}=1_{D_{N}} \otimes(1-f) \in D_{N+1} \subset D .
$$

To prove (4), start by observing that $v_{n}+v_{n}^{*}$ is a unitary of order 2 such that $\operatorname{Ad}\left(v_{n}+v_{n}^{*}\right)$ exchanges $p_{n}$ and $q_{n}$. So we can write the map $\psi_{n}$ of Lemma 2.1 as the composite of the maps

$$
\rho_{n}: M_{t(n-1)} \oplus M_{t(n-1)} \rightarrow M_{t(n)} \quad \text { and } \quad \sigma_{n}: M_{t(n)} \rightarrow M_{t(n)} \oplus M_{t(n)}
$$

given by $\rho_{n}(a, b)=a \otimes p_{n}+b \otimes q_{n}$ for $a, b \in M_{t(n-1)}$ and

$$
\sigma_{n}(a)=\left(a,\left[1 \otimes\left(v_{n}+v_{n}^{*}\right)\right] a\left[1 \otimes\left(v_{n}+v_{n}^{*}\right)\right]\right)
$$

for $a \in M_{t(n)}$. Then $D$ is the direct limit of the system

$$
M_{t(1)} \stackrel{\rho_{2} \circ \sigma_{1}}{\longrightarrow} M_{t(2)} \stackrel{\rho_{3} \circ \sigma_{2}}{\longrightarrow} M_{t(3)} \stackrel{\rho_{4} \circ \sigma_{3}}{\longrightarrow} \cdots,
$$

which is obviously a UHF algebra. 
We complete the proof by showing that (5) and (6) both imply (3), which we do by contradiction. Assume (3) fails. Choose $N_{0}$ such that $\operatorname{rank}\left(p_{n}\right)>\operatorname{rank}\left(q_{n}\right)$ for all $n \geq N_{0}$. Identifying $K_{0}\left(C^{*}\left(\mathbb{Z}_{2}, M_{t\left(N_{0}\right)}\right)\right)$ with $\mathbb{Z}^{2}$ as in Lemma 2.1(2), set $\eta_{N_{0}}=(1,-1) \in K_{0}\left(C^{*}\left(\mathbb{Z}_{2}, M_{t\left(N_{0}\right)}\right)\right)$. Let $N>N_{0}$. Using Lemma 2.3(1), we get

$$
T_{N} T_{N-1} \cdots T_{N_{0}+1}=\left(\begin{array}{cc}
\operatorname{rank}\left(e_{N}\right) & \operatorname{rank}\left(f_{N}\right) \\
\operatorname{rank}\left(f_{N}\right) & \operatorname{rank}\left(e_{N}\right)
\end{array}\right)
$$

for projections $e_{N}, f_{N} \in \bigotimes_{n=N_{0}+1}^{N} M_{k(n)}$ with $e_{N}+f_{N}=1$. Moreover, $\operatorname{rank}\left(e_{N}\right)>$ $\operatorname{rank}\left(f_{N}\right)$ by Lemma 2.3(3). Lemma 2.1(2) implies that the image $\eta_{N}$ of $\eta_{N_{0}}$ in $K_{0}\left(C^{*}\left(\mathbb{Z}_{2}, M_{t(N)}\right)\right)$ is $\left(l_{N},-l_{N}\right)$ with $l_{N}=\operatorname{rank}\left(e_{N}\right)-\operatorname{rank}\left(f_{N}\right)$. Since $l_{N} \neq 0$ for all $N$, the image $\eta$ of $\eta_{N_{0}}$ in $K_{0}\left(C^{*}\left(\mathbb{Z}_{2}, D, \alpha\right)\right)$ is nonzero. Lemma 2.12) implies that $\widehat{\alpha}_{*}(\eta)=-\eta$. Since $K_{0}\left(C^{*}\left(\mathbb{Z}_{2}, D, \alpha\right)\right)$ has no torsion, we have $-\eta \neq \eta$, so (6) fails. Moreover, for every $N \geq N_{0}$, neither $\eta_{N}>0$ nor $-\eta_{N}>0$. Therefore neither $\eta>0$ nor $-\eta>0$, whence (5) fails.

The last statement in the following proposition is a generalization of a statement at the end of Example 3.14 of [12. It is also in Example III.4 of [10].

Proposition 2.5. Let $\alpha \in \operatorname{Aut}(D)$ be a product type automorphism of order 2 as in Lemma 2.1, and adopt Notation 2.2. Then the following are equivalent:

(1) The action $\alpha$ has the tracial Rokhlin property.

(2) The dual action $\widehat{\alpha}$ is tracially approximately representable.

(3) $\Lambda(m, \infty)=0$ for all $m$.

(4) $C^{*}\left(\mathbb{Z}_{2}, D, \alpha\right)$ has a unique tracial state.

(5) $\widehat{\alpha}$ is trivial on $T\left(C^{*}\left(\mathbb{Z}_{2}, D, \alpha\right)\right)$.

(6) If $\pi$ is the Gelfand-Naimark-Segal representation associated with the unique tracial state $\tau$ on $D$, then the automorphism $\bar{\alpha}$ of $\pi(D)^{\prime \prime}$ induced by $\alpha$ is outer.

Moreover, if these conditions fail, then $C^{*}\left(\mathbb{Z}_{2}, D, \alpha\right)$ has exactly two extreme tracial states, which are interchanged by $\widehat{\alpha}$.

Proof. That (1) implies (2) is Theorem 1.6(1). To see that (2) implies (5), observe that $\widehat{\alpha}$ is tracially approximately inner by Remark 1.7. and apply Proposition 6.1 of [27]. That (4) implies (5) is obvious. For the reverse, it is evident from the description of the dual action on the direct system of crossed products $C^{*}\left(\mathbb{Z}_{2}, D_{n}\right)$ in Lemma 2.1 that $C^{*}\left(\mathbb{Z}_{2}, D, \alpha\right)$ has only one $\widehat{\alpha}$-invariant tracial state. The equivalence of (11) and (6) follows from Theorem 5.5 of [6].

We now prove that (5) implies (3), and also prove the last statement. Assume (3) fails.

Let $\operatorname{tr}_{m}$ denote the normalized trace on $M_{m}$. Tracial states on $C^{*}\left(\mathbb{Z}_{2}, D, \gamma\right)$ are in one to one correspondence with sequences $\left(\tau_{n}\right)_{n \in \mathbb{N}}$ of tracial states $\tau_{n}$ on $M_{t(n)} \oplus M_{t(n)}$ satisfying the compatibility conditions $\tau_{n} \circ \psi_{n}=\tau_{n-1}$ for all $n$. The tracial state $\tau_{n}$ has the form $\tau_{n}(a, b)=r_{n} \operatorname{tr}_{t(n)}(a)+s_{n} \operatorname{tr}_{t(n)}(b)$ for $r_{n}, s_{n} \in[0,1]$ with $r_{n}+s_{n}=1$. To describe the compatibility condition, for $\lambda \in \mathbb{R}$ define the matrix

$$
T(\lambda)=\frac{1}{2}\left(\begin{array}{cc}
1+\lambda & 1-\lambda \\
1-\lambda & 1+\lambda
\end{array}\right)=\left(\begin{array}{cc}
1 & 1 \\
1 & -1
\end{array}\right)^{-1}\left(\begin{array}{cc}
1 & 0 \\
0 & \lambda
\end{array}\right)\left(\begin{array}{cc}
1 & 1 \\
1 & -1
\end{array}\right) .
$$

From the second expression, we see that $T(\lambda \mu)=T(\lambda) T(\mu)$ for $\lambda, \mu \in \mathbb{R}$, and that $T(1)=1$. From the first expression, we see that $k(n) T\left(\lambda_{n}\right)$ is exactly the 
matrix $T_{n}$ of partial embedding multiplicities of $\psi_{n}$, as in Lemma 2.1(2). Thus, the compatibility condition is exactly $T\left(\lambda_{n}\right)\left(r_{n}, s_{n}\right)=\left(r_{n-1}, s_{n-1}\right)$ in $\mathbb{R}^{2}$.

For use below, we also observe that if $\lambda \in[0,1]$, if $\left(r_{0}, s_{0}\right),(r, s) \in \mathbb{R}^{2}$ satisfy $T(\lambda)\left(r_{0}, s_{0}\right)=(r, s)$, and if $r_{0}, s_{0} \in[0,1]$ with $r_{0}+s_{0}=1$, then $r, s \in[0,1]$ with $r+s=1$.

We claim that a sequence $\left(r_{n}, s_{n}\right)_{n \in \mathbb{N}}$ corresponds to a tracial state on the crossed product $C^{*}\left(\mathbb{Z}_{2}, D, \gamma\right)$ if and only if there is $r \in[0,1]$ such that for all $n \in \mathbb{N}$ we have $\left(r_{n}, s_{n}\right)=T(\Lambda(n, \infty))(r, 1-r)$. One direction is easy: given $r$, the sequence $\left(r_{n}, s_{n}\right)_{n \in \mathbb{N}}$ defined by $T(\Lambda(n, \infty))(r, 1-r)=\left(r_{n}, s_{n}\right)$ clearly satisfies $r_{n}, s_{n} \in[0,1]$ and $r_{n}+s_{n}=1$, and the compatibility condition follows from the relation $\Lambda(n, \infty) \lambda_{n}=\Lambda(n-1, \infty)$. For the other direction, choose $m \in \mathbb{N}$ such that $\Lambda(m, \infty) \neq 0$. Define $r, s \in \mathbb{R}$ by $(r, s)=T(\Lambda(m, \infty))^{-1}\left(r_{m}, s_{m}\right)$. The compatibility condition guarantees that $T(\Lambda(n, \infty))^{-1}\left(r_{n}, s_{n}\right)=(r, s)$ for all $n \geq m$. Now $\sum_{k=m+1}^{\infty} \log \left(\lambda_{k}\right)$ converges to $\log (\Lambda(m, \infty))$, so

$$
\log \left(\lim _{n \rightarrow \infty} \Lambda(n, \infty)\right)=\lim _{n \rightarrow \infty} \sum_{l=n+1}^{\infty} \log \left(\lambda_{l}\right)=0,
$$

whence $\lim _{n \rightarrow \infty} \Lambda(n, \infty)=1$. Therefore $\lim _{n \rightarrow \infty} r_{n}=r$ and $\lim _{n \rightarrow \infty} s_{n}=s$. It follows that $r, s \in[0,1]$ and $r+s=1$. We already have $T(\Lambda(n, \infty))(r, s)=\left(r_{n}, s_{n}\right)$ for $n \geq m$, and the compatibility condition implies that this relation holds for $n<m$ as well. This completes the proof of the claim.

We now have an affine parametrization of the tracial states on $C^{*}\left(\mathbb{Z}_{2}, D, \gamma\right)$ by $[0,1]$, from which it is clear that there are exactly two extreme tracial states. That the dual action exchanges them is clear from the identification of the dual action with the flip in Lemma 2.1 Thus, (5) fails. We have also verified the last claim whenever (3) fails.

We now prove that (3) implies (1). Assuming (3), we verify the hypotheses of Theorem 1.9. (Here, we use only a weak form of this theorem.) It suffices to consider finite subsets $F$ of the dense subalgebra $\bigcup_{n=1}^{\infty} D_{n} \subset D$. Thus, let $F \subset D_{N}$, and let $\varepsilon>0$. By hypothesis, we have $\Lambda(N, \infty)=0$. So there exists $n$ such that $\Lambda(N, N+n)<\varepsilon$. Using Lemma 2.3, write

$$
\bigotimes_{m=N+1}^{N+n} \operatorname{Ad}\left(p_{m}-q_{m}\right)=\operatorname{Ad}(p-q)
$$

for projections $p, q \in \bigotimes_{m=N+1}^{N+n} M_{k(m)}$ such that $p+q=1$ and $\operatorname{rank}(p) \geq \operatorname{rank}(q)$, and note that Lemma 2.3.5] implies

$$
\operatorname{rank}(p)-\operatorname{rank}(q)=\Lambda(N, N+n)[\operatorname{rank}(p)+\operatorname{rank}(q)] .
$$

Choose $v \in \bigotimes_{m=N+1}^{N+n} M_{k(m)}$ such that $v v^{*} \leq p$ and $v^{*} v=q$. Define $f_{0}, f_{1} \in$ $\bigotimes_{m=N+1}^{N+n} M_{k(m)}$ by

$$
f_{0}=\frac{1}{2}\left(v v^{*}+q+v+v^{*}\right) \quad \text { and } \quad f_{1}=\frac{1}{2}\left(v v^{*}+q-v-v^{*}\right) .
$$

Then one verifies that $f_{0}$ and $f_{1}$ are orthogonal projections with sum $v v^{*}+q$, and that moreover the unitary $w=p-q$ satisfies $w f_{0} w^{*}=f_{1}$ and $w f_{1} w^{*}=f_{0}$. Now set

$$
e_{0}=1_{D_{N}} \otimes f_{0}, e_{1}=1_{D_{N}} \otimes f_{1} \in D_{N+n} \subset D .
$$


Then $e_{0}$ and $e_{1}$ commute exactly with every element of $D_{N}$, and hence with every element of $F$, and moreover $\alpha\left(e_{0}\right)=e_{1}$ and $\alpha\left(e_{1}\right)=e_{0}$. Furthermore, the unique tracial state $\tau$ satisfies

$$
\tau\left(1-e_{0}-e_{1}\right)=1-\frac{2 \cdot \operatorname{rank}(q)}{\operatorname{rank}(p)+\operatorname{rank}(q)}=\Lambda(N, N+n)<\varepsilon .
$$

So Theorem 1.9 implies that $\alpha$ has the tracial Rokhlin property.

Proposition 2.6. Let $\alpha \in \operatorname{Aut}(D)$ be a product type automorphism of order 2 as in Lemma 2.1, and adopt Notation 2.2. Then the following are equivalent:

(1) The action of $\mathbb{Z}_{2}$ generated by $\alpha$ is outer, that is, there is no unitary $u \in D$ such that $u^{2}=1$ and $u a u^{*}=\alpha(a)$ for all $a \in D$.

(2) The automorphism $\alpha$ is outer, that is, there is no unitary $u \in D$ such that $u u^{*}=\alpha(a)$ for all $a \in D$.

(3) There are infinitely many $n \in \mathbb{N}$ such that $\lambda_{n}<1$ (that is, $q_{n} \neq 0$ ).

(4) $C^{*}\left(\mathbb{Z}_{2}, D, \alpha\right)$ is simple.

Proof. We first prove that (11) implies (2). This uses only the fact that the center of $A$ is $\mathbb{C} \cdot 1$. So suppose that $u \in D$ is a unitary such that $u a u^{*}=\alpha(a)$ for all $a \in D$. Putting $a=u$, we get $\alpha(u)=u$. Using $\alpha^{2}=\operatorname{id}_{A}$, we then find that $u^{2}$ is central. Therefore $u^{2} \in \mathbb{C} \cdot 1$. So there is $\omega \in \mathbb{C}$ such that $v=\omega u$ satisfies $v^{2}=1$, and clearly $v a v^{*}=\alpha(a)$ for all $a \in D$.

That (2) implies (4) follows from Theorem 3.1 of [17].

If (1) fails, it is well known that $C^{*}\left(\mathbb{Z}_{2}, D, \alpha\right) \cong D \oplus D$. Since $D \oplus D$ is not simple, we have proved that (4) implies (11).

It is easy to see that (11) implies (3): if (3) fails, then there is $n$ such that $q_{m}=0$ for all $m>n$, and

$$
u=\left(p_{1}-q_{1}\right) \otimes\left(p_{2}-q_{2}\right) \otimes \cdots \otimes\left(p_{n}-q_{n}\right) \otimes 1 \in D
$$

is a unitary such that $u^{2}=1$ and $u a u^{*}=\alpha(a)$ for all $a \in D$.

We prove that (3) implies (4). Using Lemma 2.3(4), we may pass to a subsystem and assume that $\operatorname{rank}\left(q_{n}\right) \neq 0$ for all $n$. In this case, Lemma 2.1 shows that the partial embedding multiplicities in the direct system of crossed products are all nonzero, whence $C^{*}\left(\mathbb{Z}_{2}, D, \alpha\right)$ is simple.

We are now ready to give examples. The first example is the most regular possible, and is given to contrast with the remaining ones.

Example 2.7. Let $\alpha$ be the automorphism of order 2 given by

$$
D=\bigotimes_{n=1}^{\infty} M_{2} \quad \text { and } \quad \alpha=\bigotimes_{n=1}^{\infty} \operatorname{Ad}\left(\begin{array}{cc}
1 & 0 \\
0 & -1
\end{array}\right) \text {. }
$$

Then the action of $\mathbb{Z}_{2}$ generated by $\alpha$ is strictly approximately representable and has the strict Rokhlin property, the crossed product is again the $2^{\infty}$ UHF algebra, and the dual action is just another copy of the given action. All this is easily proved using Proposition 2.4 (and Lemma 2.1 to precisely identify the crossed product and dual action), and is also a special case of Example 3.2 of [13].

Example 2.8. Let $\alpha$ be the automorphism of order 2 given by

$$
D=\bigotimes_{n=1}^{\infty} M_{2^{n}} \quad \text { and } \quad \alpha=\bigotimes_{n=1}^{\infty} \operatorname{Ad}\left(1_{2^{n-1}+1} \oplus\left(-1_{2^{n-1}-1}\right)\right)
$$


The automorphism in the $n$th tensor factor is conjugation by a diagonal unitary in which $2^{n-1}+1$ diagonal entries are equal to 1 and $2^{n-1}-1$ diagonal entries are equal to -1 . Note that $D$ is the $2^{\infty}$ UHF algebra.

Following Notation 2.2, we have $\lambda_{n} \neq 0$ for all $n$, but $\Lambda(n, \infty)=0$ for all $n$. Applying Lemma 2.1 and Propositions 2.4 and 2.5, we obtain the following:

(1) The action $\alpha$ has the tracial Rokhlin property but not the strict Rokhlin property.

(2) The action $\alpha$ is strictly approximately representable.

(3) The dual action $\widehat{\alpha}$ has the strict Rokhlin property.

(4) The dual action $\widehat{\alpha}$ is tracially approximately representable but not strictly approximately representable.

(5) The dual action $\widehat{\alpha}$ is nontrivial on $K_{0}\left(C^{*}\left(\mathbb{Z}_{2}, D, \alpha\right)\right)$.

(6) $D$ is a UHF algebra.

(7) $C^{*}\left(\mathbb{Z}_{2}, D, \alpha\right)$ is a simple AF algebra with unique tracial state but is not a $\mathrm{UHF}$ algebra.

The following example was suggested by Masaki Izumi. Some of the properties given here are folklore, but we have been unable to find a reference for the proofs. This example is a special case of an example used for other purposes in Example 3.14 of 12 .

Example 2.9. Let $\alpha$ be the automorphism of order 2 given by

$$
D=\bigotimes_{n=1}^{\infty} M_{2^{n}} \quad \text { and } \quad \alpha=\bigotimes_{n=1}^{\infty} \operatorname{Ad}\left(1_{2^{n}-1} \oplus(-1)\right)
$$

The automorphism in the $n$th tensor factor is conjugation by a diagonal unitary in which $2^{n}-1$ diagonal entries are equal to 1 and one diagonal entry is equal to -1 . Note that $D$ is the $2^{\infty}$ UHF algebra.

Following Notation 2.2, we have $\Lambda(n, \infty) \neq 0$ for all $n$. Applying Lemma 2.1 and Propositions 2.4, 2.5, and 2.6, we obtain the following:

(1) The action $\alpha$ is outer but does not have the tracial Rokhlin property.

(2) The action $\alpha$ is strictly approximately representable.

(3) The dual action $\widehat{\alpha}$ has the strict Rokhlin property.

(4) The dual action $\widehat{\alpha}$ is not tracially approximately representable.

(5) The dual action $\widehat{\alpha}$ is nontrivial on $T\left(C^{*}\left(\mathbb{Z}_{2}, D, \alpha\right)\right)$.

(6) $D$ is a UHF algebra.

(7) $C^{*}\left(\mathbb{Z}_{2}, D, \alpha\right)$ is a simple AF algebra with more than one tracial state.

(8) In the factor representation of $D$ associated to the trace, $\alpha$ becomes inner.

Example 2.10. Let $B$ be the $2^{\infty}$ UHF algebra, and let $D$ be a UHF algebra such that $B \otimes D \neq D$. Then there exists an action of $\mathbb{Z}_{2}$ on $D$ with the tracial Rokhlin property, but there is no action of $\mathbb{Z}_{2}$ on $D$ with the strict Rokhlin property.

For the first statement, write

$$
D=M_{m} \otimes \bigotimes_{n=2}^{\infty} M_{2 k(n)+1}
$$

with $m$ a power of 2 and with $k(n) \geq 1$ for all $n$. Then take

$$
\alpha=\operatorname{id}_{M_{m}} \otimes \bigotimes_{n=1}^{\infty} \operatorname{Ad}\left(1_{k(n)+1} \oplus\left(-1_{k(n)}\right)\right) .
$$


Following Notation 2.2, one has $\lambda_{n} \leq \frac{1}{3}$ for all $n \geq 2$. Thus, condition (3) of Proposition 2.5 is satisfied.

Now suppose $\alpha$ is any action of $\mathbb{Z}_{2}$ on $D$. Theorem 3.5 of [14 implies, in particular, that if the action had the strict Rokhlin property, then $D$ would be isomorphic to its tensor product with the $2^{\infty}$ UHF algebra, contrary to hypothesis.

\section{Blackadar's EXAmple has the tracial RoKhlin PROPERTY}

This section is devoted to the following example.

Example 3.1. Let $A$ be the $2^{\infty}$ UHF algebra, and let $\alpha$ be the automorphism constructed in Section 5 of [5]. It follows from Corollary 5.3.2 of [5] and Takai duality [30] that $C^{*}\left(\mathbb{Z}_{2}, A, \alpha\right)$ is not an AF algebra. We prove in Proposition 3.4 below that $\alpha$ generates an action of $\mathbb{Z}_{2}$ with the tracial Rokhlin property, and in Proposition 3.5 below that this action does not have the strict Rokhlin property, and that the the dual action is tracially approximately representable but its generator induces a nontrivial automorphism of $K_{1}$. By construction, the action of $\mathbb{Z}_{2}$ generated by $\alpha$ is strictly approximately representable in the sense of Definition 1.3 . (The construction is recalled below.) It follows from Theorem 1.5](2) that the dual action on $C^{*}\left(\mathbb{Z}_{2}, A, \alpha\right)$ has the strict Rokhlin property.

We remark that the methods used to prove the tracial Rokhlin property in this example seem likely to be more typical of proofs that actions on $\mathrm{AH}$ algebras have the tracial Rokhlin property than the methods used for Proposition 2.10 of [28].

We begin with a convenient description of the construction in [5], following Section 5 there. We make the convention that in any block matrix decomposition, all blocks are to be the same size, and we write $1_{n}$ for the identity of $M_{n}$. We take the identification of $M_{m} \otimes M_{n}$ with $M_{m n}$ to send $a \otimes e_{j, k}$ to the $n \times n$ block matrix with $m \times m$ blocks, of which the $(j, k)$ block is $a$ and the rest are zero. Thus, $a \otimes 1=\operatorname{diag}(a, a, \ldots, a)$. Also, we identify the circle $S^{1}$ with $\mathbb{R} / \mathbb{Z}$, and write elements of $C\left(S^{1}\right)$ as functions on $[0,1]$ whose values at 0 and 1 are equal.

Following Definition 3.1.1 of [5], we choose a standard twice around embedding $\varphi^{+}: C\left(S^{1}\right) \rightarrow C\left(S^{1}, M_{2}\right)$, given by choosing a continuous unitary path $c \in$ $C\left([0,1], M_{2}\right)$ with

$$
c(0)=1 \quad \text { and } \quad c(1)=\left(\begin{array}{cc}
0 & 1 \\
1 & 0
\end{array}\right)
$$

and then setting

$$
\varphi^{+}(f)(t)=c(t)\left(\begin{array}{cc}
f\left(\frac{1}{2} t\right) & 0 \\
0 & f\left(\frac{1}{2}(t+1)\right)
\end{array}\right) c(t)^{*}
$$

for $f \in C\left(S^{1}\right)$. Further let $\varphi^{-}: C\left(S^{1}\right) \rightarrow C\left(S^{1}, M_{2}\right)$ be the standard -2 times around embedding $\varphi^{-}(f)(t)=\varphi^{+}(f)(1-t)$. Extend everything, using the same notation, to embeddings of $C\left(S^{1}, M_{m}\right)$ in $C\left(S^{1}, M_{2 m}\right)$, by using $\varphi^{+} \otimes \mathrm{id}_{M_{m}}$, etc.

Following Section 5 of [5], set $A_{n}=C\left(S^{1}, M_{4^{n}}\right)$ and, remembering our convention on block sizes, define a unitary in $M_{4^{n}} \subset A_{n}$ by $u_{n}=\operatorname{diag}(1,-1)$. Further define $\psi_{n}: A_{n} \rightarrow A_{n+1}$ by

$$
\psi_{n}\left(\begin{array}{ll}
f_{1,1} & f_{1,2} \\
f_{2,1} & f_{2,2}
\end{array}\right)=\left(\begin{array}{cccc}
\varphi^{+}\left(f_{1,1}\right) & 0 & \varphi^{+}\left(f_{1,2}\right) & 0 \\
0 & \varphi^{-}\left(f_{2,2}\right) & 0 & \varphi^{-}\left(f_{2,1}\right) \\
\varphi^{+}\left(f_{2,1}\right) & 0 & \varphi^{+}\left(f_{2,2}\right) & 0 \\
0 & \varphi^{-}\left(f_{1,2}\right) & 0 & \varphi^{-}\left(f_{1,1}\right)
\end{array}\right)
$$


Theorem 4.1.1, Proposition 5.1.1, and Proposition 5.1.2 of [5] show that the direct limit of the $A_{n}$ using the maps $\psi_{n}$ is the $2^{\infty}$ UHF algebra $A$, and that the automorphisms $\alpha_{n}=\operatorname{Ad}\left(u_{n}\right)$ of $A_{n}$ define an automorphism $\alpha$ of $A$ of order two. It follows from Takai duality [30] and Propositions 5.2.2 and 5.4.1 of [5] that $C^{*}\left(\mathbb{Z}_{2}, A, \alpha\right)$ is isomorphic to the tensor product of $A$ and the $2^{\infty}$ Bunce-Deddens algebra.

Further let $\iota_{n}: M_{4^{n}} \rightarrow A_{n}$ be the embedding of matrices as constant functions, and define $\sigma_{n}: M_{4^{n}} \rightarrow M_{4^{n+1}}$ by

$$
\sigma_{n}\left(\begin{array}{cc}
a_{1,1} & a_{1,2} \\
a_{2,1} & a_{2,2}
\end{array}\right)=\left(\begin{array}{cccc}
a_{1,1} \otimes 1_{2} & 0 & a_{1,2} \otimes 1_{2} & 0 \\
0 & a_{2,2} \otimes 1_{2} & 0 & a_{2,1} \otimes 1_{2} \\
a_{2,1} \otimes 1_{2} & 0 & a_{2,2} \otimes 1_{2} & 0 \\
0 & a_{1,2} \otimes 1_{2} & 0 & a_{1,1} \otimes 1_{2}
\end{array}\right) .
$$

It is a consequence of the next lemma that $\iota_{n+1} \circ \sigma_{n}=\psi_{n} \circ \iota_{n}$. Moreover, we get an automorphism $\mu_{n}$ of $M_{4^{n}}$ by defining $\mu_{n}=\operatorname{Ad}\left(u_{n}\right)$, and $\iota_{n} \circ \mu_{n}=\alpha_{n} \circ \iota_{n}$.

Lemma 3.2. Let the notation be as above. Let $\varepsilon \geq 0$, let $f \in A_{n}$, and let $a \in M_{4^{n}}$. Suppose $f(t)=a$ for all $t \in[\varepsilon, 1-\varepsilon]$. Then $\psi_{n}(f)(t)=\sigma_{n}(a)$ for all $t \in[2 \varepsilon, 1-2 \varepsilon]$. In particular, $\iota_{n+1} \circ \sigma_{n}=\psi_{n} \circ \iota_{n}$.

Proof. Write

$$
f=\left(\begin{array}{ll}
f_{1,1} & f_{1,2} \\
f_{2,1} & f_{2,2}
\end{array}\right) \quad \text { and } \quad a=\left(\begin{array}{cc}
a_{1,1} & a_{1,2} \\
a_{2,1} & a_{2,2}
\end{array}\right) .
$$

Then for each $j$ and $k$, we have $f_{j, k}(t)=a_{j, k}$ for all $t \in[\varepsilon, 1-\varepsilon]$. For $t \in[2 \varepsilon, 1-2 \varepsilon]$ we therefore get

$$
\left(\begin{array}{cc}
f_{j, k}\left(\frac{1}{2} t\right) & 0 \\
0 & f_{j, k}\left(\frac{1}{2}(t+1)\right)
\end{array}\right)=\left(\begin{array}{cc}
a_{j, k} & 0 \\
0 & a_{j, k}
\end{array}\right),
$$

which commutes with $c(t)$.

In the next lemma we show, roughly, that whenever an element $f \in A_{n}=$ $C\left(S^{1}, M_{4^{n}}\right)$ is unitarily equivalent in $C\left([0,1], M_{4^{n}}\right)$, via invariant unitaries, to a function with small variation over intervals of length $\delta$, then $\psi_{n}(f)$ is unitarily equivalent in $C\left([0,1], M_{4^{n+1}}\right)$, again via invariant unitaries, to a function with small variation over intervals of length $2 \delta$.

Lemma 3.3. Let the notation be as above. Let $t \mapsto x(t)$ be a unitary element of $C\left([0,1], M_{4^{n}}\right)$ such that $\mu_{n}(x(t))=x(t)$ for every $t \in[0,1]$. Then there exists a unitary element $t \mapsto y(t)$ of $C\left([0,1], M_{4^{n+1}}\right)$ such that $\mu_{n+1}(y(t))=y(t)$ for every $t \in[0,1]$, with the property that whenever $\varepsilon>0, \delta>0$, and $f \in A_{n}$ satisfy

$$
\left\|x(s) f(s) x(s)^{*}-x(t) f(t) x(t)^{*}\right\|<\varepsilon
$$

for all $s, t \in[0,1]$ such that $|s-t|<\delta$, then

$$
\left\|y(s) \psi_{n}(f)(s) y(s)^{*}-y(t) \psi_{n}(f)(t) y(t)^{*}\right\|<\varepsilon
$$

for all $s, t \in[0,1]$ such that $|s-t|<2 \delta$.

Proof. The equation $\mu_{n}(x(t))=x(t)$ implies that we can write $x(t)=x_{1}(t) \oplus x_{2}(t)$ for unitaries $x_{1}, x_{2} \in C\left([0,1], M_{2^{2 n-1}}\right)$. For $j=1,2$ define

$$
y_{j}(t)=\left(\begin{array}{cc}
x_{j}\left(\frac{1}{2} t\right) & 0 \\
0 & x_{j}\left(\frac{1}{2}(t+1)\right)
\end{array}\right) c(t)^{*} .
$$


Then define

$$
y(t)=\operatorname{diag}\left(y_{1}(t), y_{2}(1-t), y_{2}(t), y_{1}(1-t)\right) .
$$

Evidently $y$ is a unitary in $C\left([0,1], M_{4^{n+1}}\right)$ and $\mu_{n+1}(y(t))=y(t)$ for every $t \in[0,1]$.

To verify the conclusions of the lemma, it will simplify the notation to conjugate everything by the permutation matrix

$$
w=\left(\begin{array}{llll}
1 & 0 & 0 & 0 \\
0 & 0 & 1 & 0 \\
0 & 0 & 0 & 1 \\
0 & 1 & 0 & 0
\end{array}\right)
$$

(This conjugation is also used in Section 5 of [5].) Thus, let

$$
\widetilde{\psi}(f)=w \psi_{n}(f) w^{*}=\left(\begin{array}{cccc}
\varphi^{+}\left(f_{1,1}\right) & \varphi^{+}\left(f_{1,2}\right) & 0 & 0 \\
\varphi^{+}\left(f_{2,1}\right) & \varphi^{+}\left(f_{2,2}\right) & 0 & 0 \\
0 & 0 & \varphi^{-}\left(f_{1,1}\right) & \varphi^{-}\left(f_{1,2}\right) \\
0 & 0 & \varphi^{-}\left(f_{2,1}\right) & \varphi^{-}\left(f_{2,2}\right)
\end{array}\right)
$$

let

$$
\widetilde{u}=w u_{n+1} w^{*}=\operatorname{diag}(1,-1,-1,1),
$$

let

$$
\widetilde{y}(t)=w y(t) w^{*}=\operatorname{diag}\left(y_{1}(t), y_{2}(t), y_{1}(1-t), y_{2}(1-t)\right),
$$

and similarly define $\widetilde{\mu}$, etc.

Let $\varepsilon>0, \delta>0$, and $f \in A_{n}$ be as in the hypotheses. Define

$$
g(t)=\left(\begin{array}{ll}
y_{1}(t) \varphi^{+}\left(f_{1,1}\right)(t) y_{1}(t)^{*} & y_{1}(t) \varphi^{+}\left(f_{1,2}\right)(t) y_{2}(t)^{*} \\
y_{2}(t) \varphi^{+}\left(f_{2,1}\right)(t) y_{1}(t)^{*} & y_{2}(t) \varphi^{+}\left(f_{2,2}\right)(t) y_{2}(t)^{*}
\end{array}\right),
$$

and note that

$$
\widetilde{y}(t) \widetilde{\psi}(f) \widetilde{y}(t)^{*}=\operatorname{diag}(g(t), g(1-t)) .
$$

Accordingly, it suffices to prove that if

$$
\left\|x(s) f(s) x(s)^{*}-x(t) f(t) x(t)^{*}\right\|<\varepsilon
$$

for all $s, t \in[0,1]$ such that $|s-t|<\delta$, then

$$
\|g(s)-g(t)\|<\varepsilon
$$

for all $s, t \in[0,1]$ such that $|s-t|<2 \delta$.

Let $v$ be the permutation matrix

$$
v=\left(\begin{array}{llll}
1 & 0 & 0 & 0 \\
0 & 0 & 1 & 0 \\
0 & 1 & 0 & 0 \\
0 & 0 & 0 & 1
\end{array}\right)
$$

When one calculates $v g(t) v^{*}$ by substituting the formulas for $y_{j}(t)$ and $\varphi^{+}$in the expression for $g(t)$, the factors $c(t)$ and $c(t)^{*}$ all cancel out, and the final answer is

$$
v g(t) v^{*}=\operatorname{diag}\left(x\left(\frac{1}{2} t\right) f\left(\frac{1}{2} t\right) x\left(\frac{1}{2} t\right)^{*}, x\left(\frac{1}{2}(t+1)\right) f\left(\frac{1}{2}(t+1)\right) x\left(\frac{1}{2}(t+1)\right)^{*}\right) .
$$

Since we are assuming

$$
\left\|x(s) f(s) x(s)^{*}-x(t) f(t) x(t)^{*}\right\|<\varepsilon
$$

for all $s, t \in[0,1]$ such that $|s-t|<\delta$, it is immediate that $|s-t|<2 \delta$ implies

$$
\left\|v g(s) v^{*}-v g(t) v^{*}\right\|<\varepsilon,
$$


whence also

$$
\|g(s)-g(t)\|<\varepsilon
$$

as desired.

Proposition 3.4. The automorphism $\alpha$ of Example 3.1 generates an action of $\mathbb{Z}_{2}$ with the tracial Rokhlin property.

Proof. Let the notation be as before Lemma 3.2. Let $\tau$ be the unique tracial state on $A=\lim _{\longrightarrow} A_{n}$. Define a tracial state $\tau_{n}$ on $A_{n}$ by

$$
\tau_{n}(f)=\int_{0}^{1} \operatorname{tr}_{4^{n}}(f(t)) d t
$$

where $\operatorname{tr}_{m}$ is the normalized trace on $M_{m}$. Then one checks that $\tau_{n+1} \circ \psi_{n}=\tau_{n}$ for all $n$. It follows from the uniqueness of $\tau$ that $\left.\tau\right|_{A_{n}}=\tau_{n}$ for all $n$.

We use Theorem 1.9 to verify the tracial Rokhlin property. So let $F \subset A$ be finite and let $\varepsilon>0$. Choose $m$ and a finite set $S_{0} \subset A_{m}$ such that every element of $F$ is within $\frac{1}{8} \varepsilon$ of an element of $S_{0}$.

The set $S_{0}$ is a uniformly equicontinuous set of functions from $[0,1]$ to $M_{4^{m}}$, so there is $\delta>0$ such that whenever $s, t \in[0,1]$ satisfy $|s-t|<\delta$, then

$$
\|f(s)-f(t)\|<\frac{1}{8} \varepsilon
$$

for all $f \in S_{0}$. Choose $n \in \mathbb{N}$ with $n \geq m$ and so large that $2^{n-m} \delta>1$. Apply Lemma 3.3 a total of $n-m$ times, the first time with $x(t)=1$ for all $t$, obtaining after the last application a continuous unitary path $t \mapsto z(t)$ in $C\left([0,1], M_{4^{n}}\right)$ such that $\mu_{n}(z(t))=z(t)$ for every $t \in[0,1]$. Replacing $z(t)$ by $z(0)^{*} z(t)$, we may clearly assume that $z(0)=1$. Then, in particular,

$$
\left\|z(t)^{*} f(0) z(t)-f(t)\right\|<\frac{1}{8} \varepsilon
$$

for all $t \in[0,1]$ and all $f \in S_{0}$. Recall that we identify $C\left(S^{1}, B\right)$ with the set of functions $f \in C([0,1], B)$ such that $f(0)=f(1)$. Since the fixed point algebra $A_{n}^{\alpha_{n}}=C\left(S^{1}, M_{4^{n}}\right)^{\alpha_{n}}$ is just $C\left(S^{1}, M_{4^{n}}^{\mu_{n}}\right)$, and since $M_{4^{n}}^{\mu_{n}}$ is finite dimensional, there is an $\alpha_{n}$-invariant unitary $y \in A_{n}$ such that $y(t)=z(t)$ for $t \in\left[0,1-\frac{1}{8} \varepsilon\right]$. Then for each $f \in S_{0}$, regarded as a subset of $A_{n}$, there exists $g \in A_{n}$ such that $\left\|y^{*} g y-f\right\|<\frac{1}{4} \varepsilon$ and $g(t)=f(0)$ for $t \in\left[0,1-\frac{1}{8} \varepsilon\right]$. Let $S$ be the set of all elements $g$ obtained in this way from elements of $S_{0}$. In particular, for every $a \in F$ there is $g \in S$ such that $\left\|a-y^{*} g y\right\|<\frac{1}{2} \varepsilon$.

We claim that there are orthogonal positive elements $b_{0}, b_{1} \in A_{n+1} \subset A$ such that $b_{j} g=g b_{j}$ for all $g \in S$, and such that

$$
\alpha_{n+1}\left(b_{0}\right)=b_{1}, \quad \alpha_{n+1}\left(b_{1}\right)=b_{0}, \quad 0 \leq b_{0}, b_{1} \leq 1, \quad \text { and } \quad 0 \leq \tau\left(1-b_{0}-b_{1}\right)<\varepsilon .
$$

For this purpose, it suffices to use in place of $\psi_{n}$ the unitarily equivalent homomorphism

$$
\widetilde{\psi}=w \psi_{n}(-) w^{*}: C\left(S^{1}, M_{4^{n}}\right) \rightarrow C\left(S^{1}, M_{4^{n+1}}\right)
$$

in the proof of Lemma 3.3 (called $\omega_{n}$ in the proof of Proposition 5.1.1 of [5]), and to use in place of $\alpha_{n+1}$ the automorphism

$$
\widetilde{\alpha}=\operatorname{Ad}(w) \circ \alpha_{n+1}=\operatorname{Ad}(\widetilde{u})=\operatorname{Ad}(\operatorname{diag}(1,-1,-1,1)) .
$$


Note that this change does not require any change in the formula for the trace $\tau_{n+1}$, and also does not affect the first part of the conclusion of Lemma 3.2. Accordingly, if

$$
g=\left(\begin{array}{ll}
g_{1,1} & g_{1,2} \\
g_{2,1} & g_{2,2}
\end{array}\right) \in S,
$$

then $\widetilde{\psi}(g) \in C\left(S^{1}, M_{4^{n+1}}\right)$ satisfies

$$
\widetilde{\psi}(g)(t)=\left(\begin{array}{cccc}
g_{1,1}(0) & g_{1,2}(0) & 0 & 0 \\
g_{2,1}(0) & g_{2,2}(0) & 0 & 0 \\
0 & 0 & g_{1,1}(0) & g_{1,2}(0) \\
0 & 0 & g_{2,1}(0) & g_{2,2}(0)
\end{array}\right)=\left(\begin{array}{cc}
g(0) & 0 \\
0 & g(0)
\end{array}\right)
$$

for $t \in\left[\frac{1}{4} \varepsilon, 1-\frac{1}{4} \varepsilon\right]$.

Now set

$$
p_{0}=\frac{1}{2}\left(\begin{array}{cccc}
1 & 0 & 1 & 0 \\
0 & 1 & 0 & 1 \\
1 & 0 & 1 & 0 \\
0 & 1 & 0 & 1
\end{array}\right) \quad \text { and } \quad p_{1}=\frac{1}{2}\left(\begin{array}{cccc}
1 & 0 & -1 & 0 \\
0 & 1 & 0 & -1 \\
-1 & 0 & 1 & 0 \\
0 & -1 & 0 & 1
\end{array}\right)
$$

both in $M_{4}\left(M_{4^{n}}\right)$. In $2 \times 2$ block form, we can write

$$
\begin{gathered}
\tilde{u}=\left(\begin{array}{cc}
s & 0 \\
0 & -s
\end{array}\right) \text { with } s=\left(\begin{array}{cc}
1 & 0 \\
0 & -1
\end{array}\right), \\
p_{0}=\frac{1}{2}\left(\begin{array}{ll}
1 & 1 \\
1 & 1
\end{array}\right), \quad \text { and } \quad p_{1}=\frac{1}{2}\left(\begin{array}{cc}
1 & -1 \\
-1 & 1
\end{array}\right) .
\end{gathered}
$$

With these formulas, it is easy to check that $p_{0}$ and $p_{1}$ are projections with $p_{0}+p_{1}=$ 1 , that $p_{0}$ and $p_{1}$ commute with $\widetilde{\psi}(g)(t)$ for every $g \in S$ and $t \in\left[\frac{1}{4} \varepsilon, 1-\frac{1}{4} \varepsilon\right]$, and that $\operatorname{Ad}(\widetilde{u})$ exchanges $p_{0}$ and $p_{1}$.

Now choose and fix a continuous function $h:[0,1] \rightarrow[0,1]$ such that $h(t)=0$ for $t \notin\left[\frac{1}{4} \varepsilon, 1-\frac{1}{4} \varepsilon\right]$ and $h(t)=1$ for $t \in\left[\frac{1}{2} \varepsilon, 1-\frac{1}{2} \varepsilon\right]$, and define $b_{j}(t)=h(t) p_{j}$ for $j=0,1$. Then $b_{0}$ and $b_{1}$ are positive elements with $b_{0}, b_{1} \leq 1$, which commute with $\widetilde{\psi}(g)$ for every $g \in S$, which satisfy $b_{0} b_{1}=0$, such that $\operatorname{Ad}(\widetilde{u})$ exchanges $b_{0}$ and $b_{1}$, and such that $0 \leq \tau_{n+1}\left(1-b_{0}-b_{1}\right)<\varepsilon$. This proves the claim above.

We return to the use of $\psi_{n+1}$, and we let $b_{0}, b_{1} \in A_{n+1} \subset A$ be as in the claim (rather than its proof). In $A_{n+1} \subset A$, define $a_{0}=y^{*} b_{0} y$ and $a_{1}=y^{*} b_{1} y$. Since $\alpha(y)=y$, it follows that $a_{0}, a_{1} \in A_{n+1} \subset A$ satisfy $a_{j} y^{*} g y=y^{*} g y a_{j}$ for all $g \in S$, and

$$
a_{0} a_{1}=0, \quad \alpha_{n+1}\left(a_{0}\right)=a_{1}, \quad 0 \leq a_{0}, a_{1} \leq 1, \quad \text { and } \quad 0 \leq \tau\left(1-a_{0}-a_{1}\right)<\varepsilon .
$$

For $a \in F$ choose $g \in S$ such that $\left\|a-y^{*} g y\right\|<\frac{1}{4} \varepsilon$. Then

$$
\left\|\left[a_{j}, a\right]\right\| \leq 2\left\|a-y^{*} g y\right\|+\left\|\left[a_{j}, y^{*} g y\right]\right\|<2\left(\frac{1}{2} \varepsilon\right)+0=\varepsilon .
$$

This completes the verification of the hypotheses of Theorem 1.9, so it follows that $\alpha$ has the tracial Rokhlin property.

Proposition 3.5. Let $\alpha \in \operatorname{Aut}(A)$ be as in Example 3.1. Then:

(1) The action of $\mathbb{Z}_{2}$ generated by $\alpha$ does not have the strict Rokhlin property.

(2) The dual action on $C^{*}\left(\mathbb{Z}_{2}, A, \alpha\right)$ has the strict Rokhlin property.

(3) The dual action is tracially approximately representable.

(4) The generating automorphism $\widehat{\alpha}$ of the dual action acts nontrivially on $K_{1}\left(C^{*}\left(\mathbb{Z}_{2}, A, \alpha\right)\right)$. 
Proof. We have already observed in Example 3.1 that $C^{*}\left(\mathbb{Z}_{2}, A, \alpha\right)$ is not AF. Therefore (1) follows from Theorem 2.2 of [27.

It is immediate from the discussion following Example 3.1 that the action of $\mathbb{Z}_{2}$ generated by $\alpha$ is strictly approximately representable in the sense of Definition 1.3 . Part (2) therefore follows from Theorem 1.5)(2).

Part (3) follows from Proposition 3.4 and Theorem 1.6.1).

It remains to prove (4). We continue to follow the notation introduced after Example 3.1. Let $B_{n}$ be the fixed point algebra

$$
A_{n}^{\alpha_{n}}=C\left(S^{1}, M_{2^{2 n-1}}\right) \oplus C\left(S^{1}, M_{2^{2 n-1}}\right) \subset C\left(S^{1}, M_{4^{n}}\right),
$$

with the embedding being as $2 \times 2$ block diagonal matrices. Let $B=\lim _{\longrightarrow} B_{n}$, which is also equal to $A^{\alpha}$. Let $\beta_{n} \in \operatorname{Aut}\left(B_{n}\right)$ be $\beta_{n}(f, g)=(g, f)$. By Proposition 5.2.2 of [5] and the preceding discussion, there is a corresponding automorphism $\beta$ of the direct limit, $A \cong C^{*}\left(\mathbb{Z}_{2}, B, \beta\right)$, and the isomorphism can be chosen so that $\alpha$ generates the dual action. By Takai duality [30], it therefore suffices to show that $\beta$ is nontrivial on $K_{1}(B)$.

Following the discussion after Corollary 5.3.2 of [5], let $v \in B_{1} \subset A_{1}=C\left(S^{1}, M_{4}\right)$ be the unitary

$$
v(t)=\operatorname{diag}\left(e^{2 \pi i t}, e^{2 \pi i t}, e^{-2 \pi i t}, e^{-2 \pi i t}\right) .
$$

As there, the image of $[v]$ in $K_{1}(B)$ is nonzero. It follows from Proposition 5.3.1 of [5] that $K_{1}(B)$ is torsion free, and one checks that $\left[\beta_{1}(v)\right]=-[v]$, so $\beta_{*}([v])=$ $-[v] \neq[v]$.

\section{TORsion In $K_{0}$ OF THE CROSSED PRODUCT}

In this section, we give two examples related to 10.11 .3 of 3 . We then use one of them to show that crossed products by actions with the tracial Rokhlin property need not preserve the property of absorbing a strongly selfabsorbing $\mathrm{C}^{*}$-algebra.

Let $A$ be the simple unital AF algebra with $K_{0}(A) \cong \mathbb{Z}\left[\frac{1}{2}\right] \oplus \mathbb{Z}$, with $\mathbb{Z}\left[\frac{1}{2}\right]$ given the order from $\mathbb{R}$, with the strict order from the first coordinate on the direct sum, and with $\left[1_{A}\right]=(1,0)$. A proof is outlined in 10.11 .3 of $[3$, that if there is an automorphism $\alpha$ of $A$ of order 2 such that $\alpha_{*}(\eta, k)=(\eta,-k)$ on $K_{0},(A)$, then $C^{*}\left(\mathbb{Z}_{2}, A, \alpha\right)$ is not AF because $K_{1}\left(C^{*}\left(\mathbb{Z}_{2}, A, \alpha\right)\right) \neq 0$ or $K_{0}\left(C^{*}\left(\mathbb{Z}_{2}, A, \alpha\right)\right)$ has torsion. Using the K-theory of any odd UHF algebra in place of $\mathbb{Z}\left[\frac{1}{2}\right]$, we give examples of actions of $\mathbb{Z}_{2}$ showing that both possibilities can occur, and which moreover have the tracial Rokhlin property. To our knowledge, no previous example was known of an action of a finite group on an AF algebra such that the K-theory of the crossed product has torsion. The fact that the actions have the tracial Rokhlin property allows us to show that the crossed products are classifiable.

Example 4.1. Let $m \in \mathbb{N}$. Define $h: S^{2 m} \rightarrow S^{2 m}$ by $h(x)=-x$, and let $\beta \in$ $\operatorname{Aut}\left(C\left(S^{2 m}\right)\right)$ be the corresponding automorphism of order 2. For $r \in \mathbb{N}$ and $b \in$ $S^{2 m}$, define $\psi_{r, b}: C\left(S^{2 m}\right) \rightarrow M_{2 r+1} \otimes C\left(S^{2 m}\right)$ by

$$
\psi_{r, b}(f)(x)=\operatorname{diag}(f(x), f(b), f(h(b)), f(b), f(h(b)), \ldots, f(b), f(h(b)))
$$

for $x \in S^{2 m}$, where $f(b)$ and $f(h(b))$ each occur $r$ times. Choose a dense sequence $(x(n))_{n \in \mathbb{N}}$ in $S^{2 m}$, and choose a sequence $(r(n))_{n \in \mathbb{N}}$ of positive integers such that $\lim _{n \rightarrow \infty} r(n)=\infty$. (The requirement that $\lim _{n \rightarrow \infty} r(n)=\infty$ is for convenience in 
the proof of Proposition 4.2, Presumably the same results hold merely assuming $r(n) \geq 1$ for all $n$.) Set

$$
s(n)=[2 r(1)+1][2 r(2)+1] \cdots[2 r(n)+1],
$$

and set $A_{n}=M_{s(n)} \otimes C\left(S^{2 m}\right)$, which, when appropriate, we think of as

$$
M_{2 r(1)+1} \otimes M_{2 r(2)+1} \otimes \cdots \otimes M_{2 r(n)+1} \otimes C\left(S^{2 m}\right) .
$$

Define $\varphi_{n}: A_{n-1} \rightarrow A_{n}$ by $\varphi_{n}=\operatorname{id}_{M_{s(n-1)}} \otimes \psi_{r(n), x(n)}$. Then set $A=\lim _{\longrightarrow} A_{n}$.

For $r \in \mathbb{N}$ define a unitary $w_{r} \in M_{2 r+1}$ by

$$
w_{r}=\operatorname{diag}\left(1,\left(\begin{array}{ll}
0 & 1 \\
1 & 0
\end{array}\right),\left(\begin{array}{ll}
0 & 1 \\
1 & 0
\end{array}\right), \ldots,\left(\begin{array}{ll}
0 & 1 \\
1 & 0
\end{array}\right)\right) .
$$

Then define an automorphism $\alpha_{n} \in \operatorname{Aut}\left(A_{n}\right)$ of order 2 by

$$
\alpha_{n}=\operatorname{Ad}\left(w_{r(1)} \otimes w_{r(2)} \otimes \cdots \otimes w_{r(n)}\right) \otimes \beta .
$$

One checks that $\varphi_{n} \circ \alpha_{n-1}=\alpha_{n} \circ \varphi_{n}$, so that the automorphisms $\alpha_{n}$ define an automorphism $\alpha \in \operatorname{Aut}(A)$ of order 2. Define $B_{n}=C^{*}\left(\mathbb{Z}_{2}, A_{n}, \alpha_{n}\right)$.

Proposition 4.2. Let $A$ and $\alpha \in \operatorname{Aut}(A)$ be as in Example 4.1. Set $D=$ $\bigotimes_{n=1}^{\infty} M_{2 r(n)+1}$, and identify $K_{0}(D)$ with a subgroup of $\mathbb{R}$ via the map induced by the tracial state on $D$. Then:

(1) $A$ is a simple unital AF algebra with a unique tracial state.

(2) $C^{*}\left(\mathbb{Z}_{2}, A, \alpha\right)$ is a simple unital AH algebra with no dimension growth, tracial rank zero, and a unique tracial state.

(3) The action of $\mathbb{Z}_{2}$ generated by $\alpha$ has the tracial Rokhlin property but not the strict Rokhlin property.

(4) The action of $\mathbb{Z}_{2}$ generated by $\alpha$ is tracially approximately representable but not strictly approximately representable.

(5) There is a dense subgroup $G$ of $\mathbb{R}$, contained in $\mathbb{Q}$, such that $K_{0}(A) \cong G \oplus \mathbb{Z}$, with the strict order from the first coordinate, and $\alpha_{*}(\eta, k)=(\eta,-k)$ for $\eta \in G$ and $k \in \mathbb{Z} .\left(\right.$ In fact, $G=K_{0}(D)$.)

(6) The torsion subgroup of $K_{0}\left(C^{*}\left(\mathbb{Z}_{2}, A, \alpha\right)\right.$ ) is isomorphic to $\mathbb{Z}_{2^{m}}$. (In fact, $K_{0}\left(C^{*}\left(\mathbb{Z}_{2}, A, \alpha\right)\right) \cong K_{0}(D) \oplus \mathbb{Z}_{2^{m}}$, with the strict order from the first coordinate and with [1] corresponding to $(1,0)$.) Moreover, $K_{1}\left(C^{*}\left(\mathbb{Z}_{2}, A, \alpha\right)\right)=0$.

Proof. We continue using the notation of Example 4.1. The $\mathrm{C}^{*}$-algebra $A$ is almost of the form considered in [9], the only difference being that each map in the direct system uses point evaluations at two different points rather than just one. However, most of the proofs are still valid. In particular, $A$ is simple (as in Lemma 1 of [9]), and $A$ has real rank zero (by the same proof as for Theorem 9 of [9]). In addition, the K-theory computation of Theorem 13 of $[9$ is still valid. We conclude that $K_{1}(A)=0$. Moreover, using the fact that the reduced $K^{0}$-group $\widetilde{K}^{0}\left(S^{2 m}\right)$ is $\mathbb{Z}$, we find that $K_{0}(A) \cong K_{0}(D) \oplus \mathbb{Z}$. The order unit is ([1],0), and the order is the strict order from the first coordinate. That is,

$$
K_{0}(A)_{+}=\left\{(\eta, k) \in K_{0}(D) \oplus \mathbb{Z}: \eta>0\right\} \cup\{(0,0)\} .
$$

Lin's classification theorem (Theorem 5.2 of [19]), in the form given in Proposition 3.7 of [28], therefore applies to this direct limit, and we conclude that $A$ is an AF algebra. Note that it has a unique tracial state $\tau$. Since $\beta$ is multiplication by -1 on $\widetilde{K}^{0}\left(S^{2 m}\right)$ (it reverses the sign of the Bott element), it follows that, with 
$K_{0}(A)$ as described above, $\alpha_{*}(\eta, k)=(\eta,-k)$ for $\eta \in K_{0}(D)$ and $k \in \mathbb{Z}$. We have proved (1) and (5).

We show that the action of $\mathbb{Z}_{2}$ generated by $\alpha$ has the tracial Rokhlin property, which is the first part of (3). By Lemma 1.8, it suffices to show that for every $n$ and every $\varepsilon>0$, there is a projection $e \in A$ such that $e \alpha(e)=0, e$ and $\alpha(e)$ commute with every element of $A_{n}$, and $\tau(1-e-\alpha(e))<\varepsilon$. Choose $k \geq n+1$ such that $1 /[2 r(k)+1]<\varepsilon$, take

$$
e_{0}=\operatorname{diag}\left(0,\left(\begin{array}{ll}
1 & 0 \\
0 & 0
\end{array}\right),\left(\begin{array}{ll}
1 & 0 \\
0 & 0
\end{array}\right), \ldots,\left(\begin{array}{ll}
1 & 0 \\
0 & 0
\end{array}\right)\right) \in M_{2 r(k)+1},
$$

and take

$$
e=1_{M_{s(k-1)}} \otimes e_{0} \otimes 1_{C\left(S^{2 m}\right)} \in A_{k} .
$$

We now compute the crossed product. Since the action of $\mathbb{Z}_{2}$ on $S^{2 m}$ is free, and $S^{2 m} / \mathbb{Z}_{2}$ is the real projective space $\mathbb{R} P^{2 m}$, there is a strong Morita equivalence between the fixed point algebra $C\left(S^{2 m}\right)^{\mathbb{Z}_{2}}=C\left(\mathbb{R} P^{2 m}\right)$ and the crossed product $B=C^{*}\left(\mathbb{Z}_{2}, S^{2 m}, h\right)$. (For example, see Situation 2 in [29]. Our case is exactly the specific example described there.) So $C^{*}\left(\mathbb{Z}_{2}, A, \alpha\right)$ is an AH algebra with no dimension growth, which is the first part of (2). Tracial rank zero follows from Theorem 2.6 of [27], and simplicity follows from Corollary 1.6 of [27]. Because $A$ has a unique tracial state, Proposition 5.7 of [6] implies that $C^{*}\left(\mathbb{Z}_{2}, A, \alpha\right)$ has a unique tracial state.

We have $K_{*}(B) \cong K^{*}\left(\mathbb{R} P^{2 m}\right)$. Thus (see Proposition 2.7.7 of [2]), $K_{1}(B)=0$ and $K_{0}(B) \cong \mathbb{Z} \oplus \mathbb{Z}_{2^{m}}$. Moreover, for any unital homomorphism $\varepsilon: B \rightarrow M_{2}$, the isomorphism identifies $\varepsilon_{*}: \mathbb{Z} \oplus \mathbb{Z}_{2^{m}} \rightarrow \mathbb{Z}$ with the projection to the first summand.

Fix $n$, and set $y=w_{r(1)} \otimes w_{r(2)} \otimes \cdots \otimes w_{r(n-1)}$. Then $\alpha_{n-1}=\operatorname{Ad}(y) \otimes \beta$ and $\alpha_{n}=\operatorname{Ad}(y) \otimes \operatorname{Ad}\left(w_{r}\right) \otimes \beta$. The actions of $\mathbb{Z}_{2}$ which these automorphisms generate are exterior equivalent to those generated by $\operatorname{id}_{M_{s(n-1)}} \otimes \beta$ and $\operatorname{id}_{M_{s(n-1)}} \otimes \operatorname{Ad}\left(w_{r}\right) \otimes \beta$, in a manner which respects $\varphi_{n}$. Since crossed products by exterior equivalent actions are naturally isomorphic (see, for example, the proof of Theorem 2.8.3(5) of [24]), we may identify the crossed product map $\bar{\varphi}_{n}: B_{n-1} \rightarrow B_{n}$ with the tensor product $\operatorname{id}_{M_{s(n-1)}} \otimes \bar{\psi}_{r(n), x(n)}$ of $\operatorname{id}_{M_{s(n-1)}}$ and the map

$$
\bar{\psi}_{r(n), x(n)}: B \rightarrow C^{*}\left(\mathbb{Z}_{2}, M_{2 r(n)+1} \otimes C\left(S^{2 m}\right), \operatorname{Ad}\left(w_{r}\right) \otimes \beta\right)
$$

on crossed products induced by $\psi_{r(n), x(n)}$. The codomain of $\bar{\psi}_{r(n), x(n)}$ is $M_{2 r(n)+1} \otimes$ $B$ by exterior equivalence. Set

$$
w=\left(\begin{array}{ll}
0 & 1 \\
1 & 0
\end{array}\right)
$$

Let $\varepsilon: C\left(S^{2 m}\right) \rightarrow M_{2}(\mathbb{C})$ be $\varepsilon(f)=\operatorname{diag}(f(x(n)), f(h(x(n))))$, let $\iota: M_{2} \rightarrow M_{2} \otimes$ $C\left(S^{2 m}\right)$ be $a \mapsto a \otimes 1$, let $\mathbb{Z}_{2}$ act on $M_{2}$ via $\operatorname{Ad}(w)$, and let $\bar{\varepsilon}$ and $\bar{\iota}$ be the induced maps on crossed products. Using naturality, we can identify $\bar{\psi}_{r(n), x(n)}$ with

$$
a \mapsto \operatorname{diag}(a, \bar{\iota} \circ \bar{\varepsilon}(a), \bar{\iota} \circ \bar{\varepsilon}(a), \ldots, \bar{\iota} \circ \bar{\varepsilon}(a)),
$$

with $\bar{\iota} \circ \bar{\varepsilon}(a)$ occurring $r(n)$ times. We have $C^{*}\left(\mathbb{Z}_{2}, M_{2}, \operatorname{Ad}(w)\right) \cong M_{2} \oplus M_{2}$, and the map $\bar{\varepsilon}$ is unital. Identify $K_{0}\left(M_{2} \oplus M_{2}\right)$ with $\mathbb{Z} \oplus \mathbb{Z}$ in the obvious way. Identify $K_{0}(B)$ with $\mathbb{Z} \oplus \mathbb{Z}_{2^{m}}$ as above, and recall our analysis of the K-theory of unital homomorphisms $B \rightarrow M_{2}$. Then we have $\bar{\varepsilon}_{*}(k, l)=(k, k)$ for $k \in \mathbb{Z}$ and $l \in \mathbb{Z}_{2^{m}}$. 
Putting the summands together, we find that $\left(\bar{\psi}_{r(n), x(n)}\right)_{*}(k, l)=((2 r(n)+1) k, l)$. It follows that

$$
K_{0}\left(C^{*}\left(\mathbb{Z}_{2}, A, \alpha\right)\right) \cong K_{0}(D) \oplus \mathbb{Z}_{2^{m}} \quad \text { and } \quad K_{1}\left(C^{*}\left(\mathbb{Z}_{2}, A, \alpha\right)\right)=0 .
$$

The $K_{0}$-class of the identity corresponds to $\left(\left[1_{D}\right], 0\right)$. To get the order, we observe that $C^{*}\left(\mathbb{Z}_{2}, A, \alpha\right)$ has a tracial state, that the order on projections over $C^{*}\left(\mathbb{Z}_{2}, A, \alpha\right)$ is determined by traces, and that the only group homomorphism from $K_{0}(D) \oplus \mathbb{Z}_{2^{m}}$ to $\mathbb{R}$ which sends $\left(\left[1_{D}\right], 0\right)$ to 1 is the one that sends $(k, l)$ to $k$. (Recall that we are identifying $K_{0}(D)$ with its image in $\mathbb{R}$.) We have proved ([6). Since $C^{*}\left(\mathbb{Z}_{2}, A, \alpha\right)$ is now clearly not AF, an application of Theorem 2.2 of [27] shows that the action of $\mathbb{Z}_{2}$ generated by $\alpha$ does not have the strict Rokhlin property, which completes the proof of (3).

It remains to prove (4). We claim that the map $K_{0}(A) \rightarrow K_{0}\left(C^{*}\left(\mathbb{Z}_{2}, A, \alpha\right)\right)$ is not injective. It is clear that all elements $(\eta, 0) \in K_{0}(D) \oplus \mathbb{Z}_{2^{m}}$, with $\eta>0$, are positive in $K_{0}\left(C^{*}\left(\mathbb{Z}_{2}, A, \alpha\right)\right)$. The infinitesimals of $K_{0}\left(C^{*}\left(\mathbb{Z}_{2}, A, \alpha\right)\right)$ are therefore contained in $0 \oplus \mathbb{Z}_{2^{m}}$. Since infinitesimals map to infinitesimals, and the summand $0 \oplus \mathbb{Z} \subset K_{0}(D) \oplus \mathbb{Z} \cong K_{0}(A)$ consists of infinitesimals, the claim follows. Since $A$ is the fixed point algebra of $C^{*}\left(\mathbb{Z}_{2}, A, \alpha\right)$ under the dual action $\widehat{\alpha}$, Theorem 3.13 of [13] implies that $\widehat{\alpha}$ does not have the strict Rokhlin property. So, by Theorem 1.5)(2), the action generated by $\alpha$ is not strictly approximately representable.

On the other hand, $\alpha$ is strongly tracially approximately inner by Theorem 6.6 of [27, and we know $\alpha$ generates an action with the tracial Rokhlin property, so Theorem 4.6 of 27] implies that this action is tracially approximately representable.

Except for the fact that we have the K-theory of an odd UHF algebra in place of $\mathbb{Z}\left[\frac{1}{2}\right]$, we have constructed an automorphism of order 2 of the type suggested in 10.11 .3 of 3 .

Remark 4.3. We continue with the notation of Example 4.1. We saw in the proof of Proposition 4.2 that Lin's classification theorem (Theorem 5.2 of [19]) applies. (We could also use the classification theorem of [7, without appealing to the tracial Rokhlin property.) It follows, for example, that $C^{*}\left(\mathbb{Z}_{2}, A, \alpha\right)$ is stably isomorphic to the direct limit of a system of the form

$$
C\left(\mathbb{R} P^{2 m}\right) \longrightarrow M_{2 r(1)+1} \otimes C\left(\mathbb{R} P^{2 m}\right) \longrightarrow M_{(2 r(1)+1)(2 r(2)+1)} \otimes C\left(\mathbb{R} P^{2 m}\right) \longrightarrow \cdots,
$$

using one copy of the identity map and $2 r(n)$ point evaluations at the $n$th stage.

Remark 4.4. It follows from Theorems 1.5 and 1.6 that the dual of the action in Example 4.1 also has the tracial Rokhlin property but not the strict Rokhlin property, and is tracially approximately representable but not strictly approximately representable.

Example 4.5. This example is the same as Example 4.1 except that we use a different homeomorphism $h$, namely $h\left(x_{0}, x_{1}, \ldots, x_{2 m}\right)=\left(-x_{0}, x_{1}, \ldots, x_{2 m}\right)$. We choose our dense sequence to be disjoint from the fixed points of $h$; this is possible because the complement of the fixed point set is a dense open set. We define the maps $\psi_{r, b}, \varphi_{n}, \beta$, and $\alpha_{n}$ by the same formulas as in Example 4.1. Let $A$ be the direct limit, as before, and let $\alpha$ be the automorphism of order 2 obtained as the direct limit of the automorphisms $\alpha_{n}$. 
Proposition 4.6. Let $A$ and $\alpha \in \operatorname{Aut}(A)$ be as in Example 4.5. Set $D=$ $\bigotimes_{n=1}^{\infty} M_{2 r(n)+1}$, and identify $K_{0}(D)$ with a subgroup of $\mathbb{R}$ via the map induced by the tracial state on $D$. Then:

(1) A is a simple unital AF algebra with a unique tracial state.

(2) $C^{*}\left(\mathbb{Z}_{2}, A, \alpha\right)$ is a simple unital AH algebra with no dimension growth, tracial rank zero, and a unique tracial state.

(3) The action of $\mathbb{Z}_{2}$ generated by $\alpha$ has the tracial Rokhlin property but not the strict Rokhlin property.

(4) The action of $\mathbb{Z}_{2}$ generated by $\alpha$ is tracially approximately representable but not strictly approximately representable.

(5) There is a dense subgroup $G$ of $\mathbb{R}$, contained in $\mathbb{Q}$, such that $K_{0}(A) \cong G \oplus \mathbb{Z}$, with the strict order from the first coordinate, and $\alpha_{*}(\eta, k)=(\eta,-k)$ for $\eta \in G$ and $k \in \mathbb{Z} .\left(\right.$ In fact, $G=K_{0}(D)$.)

(6) $K_{0}\left(C^{*}\left(\mathbb{Z}_{2}, A, \alpha\right)\right)$ has no torsion, and $K_{1}\left(C^{*}\left(\mathbb{Z}_{2}, A, \alpha\right)\right) \cong \mathbb{Z}$.

Proof. The algebra $A$ is the same as in Proposition 4.2. so (1) and the first statement in (5) follow from the corresponding parts of Proposition 4.2, The rest of (5), the fact that $\alpha$ generates an action with the tracial Rokhlin property, and the first part of (4) all have the same proofs as the corresponding parts of Proposition 4.2.

The computation of the crossed products is now different. Set

$$
E=\left\{\left(x_{0}, x_{1}, \ldots, x_{2_{m}}\right) \in S^{2 m}: x_{0}=0\right\}
$$

and

$$
L=\left\{\left(x_{0}, x_{1}, \ldots, x_{2_{m}}\right) \in S^{2 m}: x_{0} \geq 0\right\} .
$$

Note that $E \cong S^{2 m-1}$. Let $B=C^{*}\left(\mathbb{Z}_{2}, S^{2 m}, h\right)$, as in the proof of Proposition 4.2 but using the homeomorphism $h$ of Example 4.5. Then there is an injective homomorphism $\mu: B \rightarrow C\left(L, M_{2}\right)$ which sends $f \in C\left(S^{2 m}\right)$ to the function $\mu(f)(x)=\operatorname{diag}(f(x), f(h(x)))$ and the standard implementing unitary in the crossed product to the constant function with value

$$
\left(\begin{array}{ll}
0 & 1 \\
1 & 0
\end{array}\right)
$$

Its image is

$$
\left\{f \in C\left(L, M_{2}\right): f(x)_{1,1}=f(x)_{2,2} \text { and } f(x)_{2,1}=f(x)_{1,2} \text { for } x \in E\right\} .
$$

Conjugating by the unitary

we identify $B$ with

$$
\frac{1}{\sqrt{2}}\left(\begin{array}{cc}
1 & 1 \\
-1 & 1
\end{array}\right)
$$

$$
\left\{f \in C\left(L, M_{2}\right): f(x) \text { is diagonal for } x \in E\right\} .
$$

We compute $K_{*}(B)$ via a trick. Set

$$
e=\left(\begin{array}{ll}
1 & 0 \\
0 & 0
\end{array}\right) \quad \text { and } \quad C=\left\{f \in C\left(L, M_{2}\right): f(x) \in \mathbb{C} e \text { for } x \in E\right\}
$$

The map $C(L) \rightarrow C$, sending $f \in C(L)$ to the function $x \rightarrow f(x) e$, is an isomorphism on K-theory, essentially because $K \otimes C \cong K \otimes C(L)$. Thus, $K_{0}(C) \cong \mathbb{Z}$, generated by the class of the constant function $\bar{e}$ with value $e$, so $K_{0}(C) \rightarrow K_{0}(B)$ is injective. There is a short exact sequence

$$
0 \longrightarrow C \longrightarrow B \longrightarrow C\left(S^{2 m-1}\right) \longrightarrow 0,
$$


and the associated six term exact sequence in K-theory is

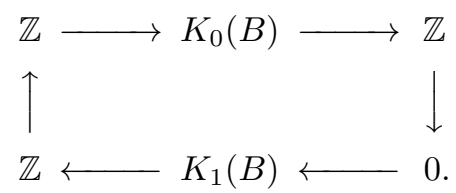

We have just seen that the map $\mathbb{Z} \rightarrow K_{0}(B)$ is injective, and $K_{0}\left(C\left(S^{2 m-1}\right)\right) \cong \mathbb{Z}$, generated by the class of the identity. So $K_{0}(B) \cong \mathbb{Z}^{2}$, generated by $[\bar{e}]$ and $[1-\bar{e}]$, and $K_{1}(B) \cong \mathbb{Z}$.

As in the proof of Proposition 4.2, we have $B_{n}=C^{*}\left(\mathbb{Z}_{2}, A_{n}, \alpha_{n}\right) \cong M_{s(n)} \otimes B$. Moreover, by the same reasoning as there, the map $B_{n-1} \rightarrow B_{n} \cong M_{2 r(n)+1} \otimes B_{n-1}$ is the tensor product of $\operatorname{id}_{M_{2 r(n)+1}}$ with a map unitarily equivalent to the direct sum of one copy of the identity map and some point evaluations. Therefore this map is unitarily equivalent to the direct sum of the identity map and a map which factors through a finite dimensional $\mathrm{C}^{*}$-algebra. So $K_{1}\left(B_{n-1}\right) \rightarrow K_{1}\left(B_{n}\right)$ is an isomorphism for all $n$, whence $K_{1}\left(C^{*}\left(\mathbb{Z}_{2}, A, \alpha\right)\right) \cong \mathbb{Z}$. Furthermore, $K_{0}\left(C^{*}\left(\mathbb{Z}_{2}, A, \alpha\right)\right)$ has no torsion because it is a direct limit of groups isomorphic to $\mathbb{Z}^{2}$. We now have (6). We also know that $C^{*}\left(\mathbb{Z}_{2}, A, \alpha\right)$ is not AF, so Theorem 2.2 of [27] implies that $\alpha$ does not have the strict Rokhlin property. This finishes the proof of (3).

We can also prove the second part of (4). Suppose $\alpha$ is strictly approximately representable. Then $\widehat{\alpha}$ has the tracial Rokhlin property by Theorem 1.5)(2). Using the dual action, $K_{1}\left(C^{*}\left(\mathbb{Z}_{2}, A, \alpha\right)\right) \cong \mathbb{Z}$ becomes a module over the group ring $\mathbb{Z}\left[\mathbb{Z}_{2}\right]$, with the nontrivial group element acting via the nontrivial automorphism. Theorem 3.3 of [14] implies that $K_{1}\left(C^{*}\left(\mathbb{Z}_{2}, A, \alpha\right)\right)$ is cohomologically trivial in the sense of Definition 3.1(2) of [14. But there are only two ways to make $\mathbb{Z}$ a $\mathbb{Z}\left[\mathbb{Z}_{2}\right]$-module. If the nontrivial group element acts trivially, then, in the notation of [14], we have $\operatorname{Coker}(\bar{N}) \cong \mathbb{Z}_{2}$, while if a nontrivial group element acts by multiplication by -1 , then $\operatorname{Ker}(\bar{N}) \cong \mathbb{Z}_{2}$. In either case, $K_{1}\left(C^{*}\left(\mathbb{Z}_{2}, A, \alpha\right)\right)$ is not cohomologically trivial, and this contradiction shows that $\alpha$ is not strictly approximately representable.

It remains to prove (2). It follows from Theorem 2.6 of [27] that $C^{*}\left(\mathbb{Z}_{2}, A, \alpha\right)$ has tracial rank zero. We have seen that it is a direct limit of type $\mathrm{I}^{*}$-algebras, so that it is nuclear and satisfies the Universal Coefficient Theorem. It is simple by Corollary 1.6 of [27]. Therefore Lin's classification theorem (Theorem 5.2 of [19]) applies. In the form given in Proposition 3.7 of $\left[28\right.$, it implies that $C^{*}\left(\mathbb{Z}_{2}, A, \alpha\right)$ is a simple AH algebra with real rank zero and no dimension growth. Proposition 5.7 of [6] implies that $C^{*}\left(\mathbb{Z}_{2}, A, \alpha\right)$ has a unique tracial state. This completes the proof.

Unlike in the situation of Example 4.1] the classification theorem of [7] does not apply to the direct limit, and we do not know of a classification proof which does not depend on the tracial Rokhlin property.

Remark 4.7. In Examples 4.1 and 4.5, the requirement $\lim _{n \rightarrow \infty} r(n)=\infty$ is merely for convenience in proving the tracial Rokhlin property. All features, including the tracial Rokhlin property, hold provided $r(n) \geq 1$ for all $n$. In Example4.5 (but not in Example 4.1), this increases the number of possibilities for $K_{0}\left(C^{*}\left(\mathbb{Z}_{2}, A, \alpha\right)\right)$. Even with the requirement $\lim _{n \rightarrow \infty} r(n)=\infty$, it is easy to choose $(r(n))_{n \in \mathbb{N}}$ so that $D$ is any odd UHF algebra. 
Remark 4.8. In Example 4.5, we do not need to require that all the matrix sizes be odd. For example, we could use in place of $\psi_{r(n), x(n)}$ the map which sends $f \in C\left(S^{2 m}\right)$ to

$$
x \mapsto \operatorname{diag}(f(x), f(y(n)), f(z(n)), f(h(z(n))))
$$

with $y(n), z(n) \in S^{2 m}$ chosen such that $h(y(n))=y(n)$ and $h(z(n)) \neq z(n)$. In place of $w_{r(n)}$ one uses

$$
\left(\begin{array}{llll}
1 & 0 & 0 & 0 \\
0 & 1 & 0 & 0 \\
0 & 0 & 0 & 1 \\
0 & 0 & 1 & 0
\end{array}\right) .
$$

One gets an action of $\mathbb{Z}_{2}$ on the AF algebra in 10.11.3 of [3] which induces the map there on K-theory.

This particular variation does not work in Example 4.1, because there are no fixed points.

Remark 4.9. Let $A$ be as in Remark4.8, As in 10.11 .3 of [3], there is no $\alpha \in \operatorname{Aut}(A)$ satisfying the following:

(1) $\alpha^{2}=\operatorname{id}_{A}$.

(2) $\alpha_{*}(\eta, k)=(\eta,-k)$ on $K_{0}(A)=\mathbb{Z}\left[\frac{1}{2}\right] \oplus \mathbb{Z}$.

(3) $C^{*}\left(\mathbb{Z}_{2}, A, \alpha\right)$ is $\mathrm{AF}$.

By Theorem 2.2 of [27, there is therefore no $\alpha \in \operatorname{Aut}(A)$ satisfying (11) and (2) and such that $\alpha$ generates an action of $\mathbb{Z}_{2}$ with the strict Rokhlin property. This is a less obvious nonexistence result than for, say, actions of $\mathbb{Z}_{2}$ on the $3^{\infty} \mathrm{UHF}$ algebra. However, it can also be obtained from Theorem 3.3 of [14].

The following question has been raised informally. Let $A$ be a separable infinite dimensional simple unital $\mathrm{C}^{*}$-algebra, let $G$ be a finite group, and let $\alpha: G \rightarrow$ $\operatorname{Aut}(A)$ have the tracial Rokhlin property. Let $Z$ be the Jiang-Su algebra [15. If $A$ is $Z$-stable, does it follow that $C^{*}(G, A, \alpha)$ is $Z$-stable? This question is motivated by the implications for classification of Theorem 2.6 of [27], which shows that such crossed products do preserve tracial rank zero. More generally, we can ask this question with an arbitrary strongly selfabsorbing $\mathrm{C}^{*}$-algebra $D$ (in the sense of [31]) in place of $Z$. Crossed products by actions with the strict Rokhlin property do preserve $D$-stability, by Corollary 3.4 of [11. We do not know whether crossed products by actions with the tracial Rokhlin property preserve $Z$-stability. However, a proof that they do will have to rely on special properties of $Z$. The construction of Example 4.1 can be used to give a counterexample (Example 4.11 below) for general strongly selfabsorbing $\mathrm{C}^{*}$-algebras $D$.

We should point out the following partial positive result for $Z$-stability.

Theorem 4.10 (Matui-Sato). Let $A$ be a simple separable unital nuclear $Z$-stable $C^{*}$-algebra satisfying the Universal Coefficient Theorem and whose tensor product with the universal UHF algebra has tracial rank zero. Suppose further that A has a unique tracial state. Let $\alpha: G \rightarrow \operatorname{Aut}(A)$ have the tracial Rokhlin property. Then $C^{*}(G, A, \alpha)$ is Z-stable.

Proof. The hypotheses on $A$ are exactly that it be in the class $\mathcal{C}_{0}$ in Definition 2.5 of [21]. Combine Theorem 5.5 of [6] and Theorem 5.3 of [21]. (See Definition 2.7(2) of [21] for the definition of strong outerness.) 
Presumably one only needs to require that $A$ have finitely many extreme tracial states. (This would follow from a converse to Theorem 5.1 of [21].)

Theorem 4.10 implies that there is in fact something special about $Z$-stability, because our example satisfies its hypotheses but not its conclusion with a suitable strongly selfabsorbing $\mathrm{C}^{*}$-algebra $D$ in place of $Z$.

Example 4.11. There is a strongly selfabsorbing UHF algebra $D$, a $D$-absorbing simple separable unital nuclear $\mathrm{C}^{*}$-algebra $C$ with tracial rank zero, with a unique tracial state, and which satisfies the Universal Coefficient Theorem, and an action $\gamma: \mathbb{Z}_{2} \rightarrow \operatorname{Aut}(C)$ with the tracial Rokhlin property, such that $C^{*}\left(\mathbb{Z}_{2}, C, \gamma\right)$ is not $D$-absorbing.

In Example 4.1 fix any $m \geq 1$ and choose $r(n)=\frac{1}{2}\left(3^{n}-1\right)$ for $n \in \mathbb{N}$. Let $D=\bigotimes_{n=1}^{\infty} M_{2 r(n)+1}$, as in Proposition 4.2. Then $D$ is the $3^{\infty}$ UHF algebra, which is certainly strongly selfabsorbing. Let $A$ and $\alpha: \mathbb{Z}_{2} \rightarrow \operatorname{Aut}(A)$ be as in Example 4.1. Define $C=C^{*}\left(\mathbb{Z}_{2}, A, \alpha\right)$, and let $\gamma: \mathbb{Z}_{2} \rightarrow \operatorname{Aut}(C)$ be the dual action. Then $\gamma$ has the tracial Rokhlin property by Remark 4.4. That $D$ has tracial rank zero, has a unique tracial state, and satisfies the Universal Coefficient Theorem, all follow from Proposition 4.2(2).

By Proposition $4.2(6)$, we have $K_{0}(C) \cong \mathbb{Z}\left[\frac{1}{3}\right] \oplus \mathbb{Z}_{2^{m}}$, with the strict order from the first coordinate and with [1] corresponding to $(1,0)$. Moreover, $K_{1}(C)=0$. So multiplication by 3 is bijective on $K_{*}(C)$. It is now easy to construct an isomorphism of scaled ordered groups $K_{0}(D \otimes C) \rightarrow K_{0}(C)$, and clearly $K_{1}(D \otimes C)=K_{0}(C)=0$. It follows from Proposition 4.2(2) that Lin's classification theorem (Theorem 5.2 of [19]) applies to $C$ and $D \otimes C$. We conclude that $D \otimes C \cong C$, that is, that $C$ is $D$-absorbing. So

Takai duality and Proposition 4.2(5) imply that $K_{0}\left(C^{*}\left(\mathbb{Z}_{2}, C, \gamma\right)\right) \cong \mathbb{Z}\left[\frac{1}{3}\right] \oplus \mathbb{Z}$.

$$
\mathbb{Z}\left[\frac{1}{3}\right] \otimes_{\mathbb{Z}} K_{0}\left(C^{*}\left(\mathbb{Z}_{2}, C, \gamma\right)\right) \nsucc K_{0}\left(C^{*}\left(\mathbb{Z}_{2}, C, \gamma\right)\right) .
$$

It follows that $C^{*}\left(\mathbb{Z}_{2}, C, \gamma\right)$ and $D \otimes C^{*}\left(\mathbb{Z}_{2}, C, \gamma\right)$ have nonisomorphic $K_{0}$-groups, whence $C^{*}\left(\mathbb{Z}_{2}, C, \gamma\right)$ is not $D$-absorbing.

Of course, one could use any other odd prime in place of 3 .

\section{Questions}

The tracial Rokhlin property, as given in Definition 1.2 of [27], is certainly not useful for $\mathrm{C}^{*}$-algebras with few projections, may well not be useful for $\mathrm{C}^{*}$-algebras with many projections but bad comparison theory, and may not be useful for nonsimple $\mathrm{C}^{*}$-algebras. The same is true of tracial approximate representability and tracial approximate innerness (Definition 5.1 of [27]). In this section, we give a brief discussion of what might be done for the tracial Rokhlin property. Similar considerations apply to the other properties, but we have less information on which to base any speculation.

If the $\mathrm{C}^{*}$-algebra $A$ is purely infinite, then the condition in Lemma 1.2 for an action $\alpha: G \rightarrow \operatorname{Aut}(A)$ to have the tracial Rokhlin property is vacuous, since $e_{g}=0$ for all $g \in G$ still permits condition (3) to hold. Definition 1.2 of [27] contains the following extra condition:

(4) With $e=\sum_{g \in G} e_{g}$ (as in condition (3)), we have $\|$ exe $\|>1-\varepsilon$. 
(This condition was inspired by Definition 2.1 of $[18$.) The definition we originally gave in [26] required, instead of condition (44), that for every $N \in \mathbb{N}$ one be able to choose the projections $e_{g}$ so that, in addition,

(5) With $e=\sum_{g \in G} e_{g}$ as before, there are $N$ mutually orthogonal projections $f_{1}, f_{2}, \ldots, f_{N} \leq e_{g}$, each of which is Murray-von Neumann equivalent to $1-e$.

Under either extra condition, one can force $e_{g} \neq 0$ for all $g \in G$. The proof of Lemma 1.5 of [27] then shows that $\alpha_{g}$ is outer for all $g \in G \backslash\{1\}$.

According to Theorem 1 of [22, if $A$ is a unital Kirchberg algebra (purely infinite simple separable and nuclear), and if $\alpha \in \operatorname{Aut}(A)$ has the property that $\alpha^{n}$ is outer for every $\alpha \neq 0$, then $\alpha$ has the strict Rokhlin property. For finite group actions, there are obvious K-theoretic obstructions to the strict Rokhlin property. For example, if $A$ is any unital purely infinite simple $\mathrm{C}^{*}$-algebra with $K_{0}(A) \cong \mathbb{Z}$ and generated by [1], such as $\mathcal{O}_{\infty}$, then every automorphism is trivial on $K_{0}$. If $\alpha: G \rightarrow \operatorname{Aut}(A)$ had the strict Rokhlin property, then [1] $\in K_{0}(A)$ would then be divisible by $\operatorname{card}(G)$, forcing $G$ to be trivial. For classifiable algebras there are less obvious K-theoretic obstructions, such as Theorem 3.5 of [14]. However, the following conjecture seems plausible.

Conjecture 5.1. Let $A$ be a unital Kirchberg algebra, and let $\alpha: G \rightarrow \operatorname{Aut}(A)$ be an action of a finite group $G$ on $A$ such that $\alpha_{g}$ is outer for all $g \in G \backslash\{1\}$. Then $\alpha$ has the tracial Rokhlin property, as given in Definition 1.2 of [27].

Since the proof in 22] uses nuclearity in an apparently essential way, we do not know what to expect in the nonnuclear case. For algebras which are infinite but not purely infinite, the situation is even less clear.

We now turn to algebras with few projections. The following discussion applies only to simple unital $\mathrm{C}^{*}$-algebras with "enough traces"; if, for example, the algebra is stably finite but the comparison theory of projections is bad, the smallness conditions on the leftovers must be changed.

The criterion for the tracial Rokhlin property in Theorem 1.9 does not mention projections, and thus makes sense even if there are no nontrivial projections. We suppose, for example, that it probably holds for the flip $a \otimes b \mapsto b \otimes a$ on the tensor product of two copies of the Jiang-Su algebra $Z$ [15]. (As shown in [23, for many simple $\mathrm{C}^{*}$-algebras which have many projections, the flip has the tracial Rokhlin property.) This suggests taking the criterion in Theorem 1.9 as the definition in the absence of enough projections. Since Theorem 1.9 depends on tracial rank zero, this suggestion may need to be limited to algebras which are expected to be classifiable.

There are two tests. First, one might hope for an analog of Theorem 2.6 of [27] and its generalization in 23 . To include algebras with few projections, and lacking an abstract criterion for classifiability for such algebras, we propose the following problem.

Problem 5.2. Let $A$ be an infinite dimensional simple unital $\mathrm{C}^{*}$-algebra which is isomorphic to a direct limit, with no dimension growth, of recursive subhomogeneous algebras, as in Theorem 3.6 of [25]. Let $\alpha: G \rightarrow \operatorname{Aut}(A)$ be an action of a finite group $G$ on $A$, and suppose that $\alpha$ satisfies the condition of Theorem 1.9. Does it follow that $C^{*}(G, A, \alpha)$ is again isomorphic to a direct limit, with no dimension growth, of recursive subhomogeneous algebras? 
The class contains the Jiang-Su algebra $Z$, as well as other simple unital $\mathrm{C}^{*}$ algebras with no nontrivial projections.

Of course, if the answer is no, this might mean that the class of $\mathrm{C}^{*}$-algebras is wrong, rather than indicating any problem with the proposed definition of the tracial Rokhlin property.

For the second test, we begin with the following result of Archey.

Theorem 5.3 (Theorems 4.2, 5.1, and 6.2 of [1]). Let $A$ be an infinite dimensional simple unital $C^{*}$-algebra, let $G$ be a finite group, and let $\alpha: G \rightarrow \operatorname{Aut}(A)$ be an action with the tracial Rokhlin property. Suppose that $A$ has real rank zero, stable rank one, and that the order on projections over $A$ is determined by traces (Definition 2.4 of [27]; a stable version of Blackadar's Second Fundamental Comparability Question as in 1.3.1 in [4). Then $C^{*}(G, A, \alpha)$ also has these three properties.

Theorem 5.3 motivates the following problem. We drop real rank zero, since we are interested in algebras with few projections.

Problem 5.4. Let $A$ be an infinite dimensional simple unital $\mathrm{C}^{*}$-algebra, let $G$ be a finite group, and let $\alpha: G \rightarrow \operatorname{Aut}(A)$ be an action which satisfies the condition of Theorem 1.9. Suppose that $A$ has stable rank one and that the order on projections over $A$ is determined by traces. Does $C^{*}(G, A, \alpha)$ also have these two properties?

We now turn to nonsimple $\mathrm{C}^{*}$-algebras. Ultimately, one hopes for a general theory encompassing actions on simple $\mathrm{C}^{*}$-algebras, on commutative $\mathrm{C}^{*}$-algebras, and on everything in between. It is not clear what the correct definitions are. The commutative case with many projections is not very informative, because of the following result.

Proposition 5.5. Let the finite group $G$ act on the Cantor set $X$, and let $\alpha: G \rightarrow$ Aut $(C(X))$ be the action $\alpha_{g}(f)(x)=f\left(g^{-1} x\right)$ for $x \in X, g \in G$, and $f \in C(X)$. Then the following are equivalent:

(1) $\alpha$ has the strict Rokhlin property.

(2) $\alpha$ satisfies the condition of the definition of the tracial Rokhlin property (Definition 1.2 of [27; see the beginning of this section).

(3) $\alpha$ satisfies the condition of Lemma 1.2.

(4) The action of $G$ on $X$ is free.

Proof. It is clear that (12) implies (2) and (2) implies (3).

Assume (3). Let $x \in X$. Choose a nonempty compact open set $K \subset X$ such that $K \cap G x=\varnothing$. Apply (3) with $F=\varnothing$, with $\varepsilon=\frac{1}{2}$, and with $x=\chi_{K}$, obtaining disjoint compact open sets $L_{g}$ for $g \in G$ such that $\left\|\chi_{g L_{h}}-\chi_{L_{g h}}\right\|<\frac{1}{2}$ for all $g, h \in G$, and such that, with $L=\bigcup_{g \in G} L_{g}$, we have $\chi_{X \backslash L} \precsim \chi_{K}$. It follows that $g L_{h}=L_{g h}$ for all $g, h \in G$, and that $X \backslash L \subset K$. Since $x \in L$, the map $g \mapsto g x$ is injective. Since this is true for all $x \in X$, we have proved (4).

Now assume (4). For each $x \in X$, use continuity and freeness to choose a compact open set $K_{x} \subset X$ which contains $x$ and such that the sets $g K_{x}$, for $g \in G$, are disjoint. Choose $x_{1}, x_{2}, \ldots, x_{n} \in X$ such that $K_{x_{1}}, K_{x_{2}}, \ldots, K_{x_{n}}$ cover $X$. Inductively set $N_{1}=K_{x_{1}}$ and $N_{k+1}=N_{k} \cup\left[K_{x_{k+1}} \cap\left(X \backslash G N_{k}\right)\right]$. The sets $g N_{1}$, for $g \in G$, are disjoint. Moreover, if the sets $g N_{k}$, for $g \in G$, are disjoint, then for 
distinct $g, h \in G$ we have

$$
\begin{aligned}
g N_{k+1} \cap h N_{k+1} \subset\left[g N_{k} \cap h N_{k}\right] \cup\left[g N_{k} \cap h\left(X \backslash G N_{k}\right)\right] \\
\cup\left[g\left(X \backslash G N_{k}\right) \cap h N_{k}\right] \cup\left[g K_{x_{k+1}} \cap h K_{x_{k+1}}\right] .
\end{aligned}
$$

All four terms are clearly empty. By induction, therefore, the sets $g N_{n}$, for $g \in G$, are disjoint. A related argument shows that $G N_{k}$ contains $G K_{x_{1}}, G K_{x_{2}}, \ldots, G K_{x_{n}}$, whence $G N_{n}=X$.

Now the projections $e_{g}=\chi_{g N_{n}}$ are central, satisfy $\sum_{g \in G} e_{g}=1$, and satisfy $\alpha_{g}\left(e_{h}\right)=e_{g h}$ for all $g, h \in G$. Accordingly, $\alpha$ has the strict Rokhlin property.

We suspect, however, that the condition of Lemma 1.2, or even the tracial Rokhlin property as defined here, will not be adequate to prove general theorems about actions on nonsimple $\mathrm{C}^{*}$-algebras.

\section{ACKNOWLEDGEMENTS}

The author is grateful to Masaki Izumi for discussions concerning the (strict) Rokhlin property. In particular, he suggested Example 2.9. The author would also like to thank Hiroyuki Osaka for carefully reading earlier versions of this paper, and catching a number of misprints and suggesting many improvements.

\section{REFERENCES}

[1] Dawn Archey, Crossed product $C^{*}$-algebras by finite group actions with the tracial Rokhlin property, Rocky Mountain J. Math. 41 (2011), no. 6, 1755-1768, DOI 10.1216/RMJ-201141-6-1755. MR2854735 (2012m:46074)

[2] Michael F. Atiyah, K-theory, Lecture notes by D. W. Anderson, W. A. Benjamin, Inc., New York-Amsterdam, 1967. MR0224083 (36 \#7130)

[3] Bruce Blackadar, K-theory for operator algebras, Mathematical Sciences Research Institute Publications, vol. 5, Springer-Verlag, New York, 1986. MR859867 (88g:46082)

[4] Bruce Blackadar, Comparison theory for simple $C^{*}$-algebras, Operator algebras and applications, Vol. 1, London Math. Soc. Lecture Note Ser., vol. 135, Cambridge Univ. Press, Cambridge, 1988, pp. 21-54. MR996438 (90g:46078)

[5] Bruce Blackadar, Symmetries of the CAR algebra, Ann. of Math. (2) 131 (1990), no. 3, 589-623, DOI 10.2307/1971472. MR1053492(91i:46084)

[6] Siegfried Echterhoff, Wolfgang Lück, N. Christopher Phillips, and Samuel Walters, The structure of crossed products of irrational rotation algebras by finite subgroups of $\mathrm{SL}_{2}(\mathbb{Z})$, J. Reine Angew. Math. 639 (2010), 173-221, DOI 10.1515/CRELLE.2010.015. MR.2608195 (2011c:46127)

[7] George A. Elliott, Guihua Gong, and Liangqing Li, On the classification of simple inductive limit $C^{*}$-algebras. II. The isomorphism theorem, Invent. Math. 168 (2007), no. 2, 249-320, DOI 10.1007/s00222-006-0033-y. MR2289866 (2010g:46102)

[8] Thierry Fack and O. Maréchal, Sur la classification des symétries des $C^{*}$-algebres UHF (French), Canad. J. Math. 31 (1979), no. 3, 496-523, DOI 10.4153/CJM-1979-055-7. MR.536360 (81d:46065)

[9] Kenneth R. Goodearl, Notes on a class of simple $C^{*}$-algebras with real rank zero, Publ. Mat. 36 (1992), no. 2A, 637-654 (1993), DOI 10.5565/PUBLMAT_362A92_23. MR.1209829 (94f:46092)

[10] David Handelman and Wulf Rossmann, Actions of compact groups on AF $C^{*}$-algebras, Illinois J. Math. 29 (1985), no. 1, 51-95. MR769758 (86f:46071)

[11] Ilan Hirshberg and Wilhelm Winter, Rokhlin actions and self-absorbing $C^{*}$-algebras, Pacific J. Math. 233 (2007), no. 1, 125-143, DOI 10.2140/pjm.2007.233.125. MR.2366371(2009i:46097)

[12] Masaki Izumi, Inclusions of simple $C^{*}$-algebras, J. Reine Angew. Math. 547 (2002), 97-138, DOI 10.1515/crll.2002.055. MR:1900138(2003b:46072)

[13] Masaki Izumi, Finite group actions on $C^{*}$-algebras with the Rohlin property. I, Duke Math. J. 122 (2004), no. 2, 233-280, DOI 10.1215/S0012-7094-04-12221-3. MR.2053753(2005a:46142) 
[14] Masaki Izumi, Finite group actions on $C^{*}$-algebras with the Rohlin property. II, Adv. Math. 184 (2004), no. 1, 119-160, DOI 10.1016/S0001-8708(03)00140-3. MR2047851(2005b:46153)

[15] Xinhui Jiang and Hongbing $\mathrm{Su}$, On a simple unital projectionless $C^{*}$-algebra, Amer. J. Math. 121 (1999), no. 2, 359-413. MR1680321 (2000a:46104)

[16] Akitaka Kishimoto, On the fixed point algebra of a UHF algebra under a periodic automorphism of product type, Publ. Res. Inst. Math. Sci. 13 (1977/78), no. 3, 777-791. MR0500177 (58 \#17863)

[17] Akitaka Kishimoto, Outer automorphisms and reduced crossed products of simple $C^{*}$ algebras, Comm. Math. Phys. 81 (1981), no. 3, 429-435. MR634163 (83c:46061)

[18] Huaxin Lin, Tracially AF $C^{*}$-algebras, Trans. Amer. Math. Soc. 353 (2001), no. 2, 693-722 (electronic), DOI 10.1090/S0002-9947-00-02680-5. MR.1804513 (2001j:46089)

[19] Huaxin Lin, Classification of simple $C^{*}$-algebras of tracial topological rank zero, Duke Math. J. 125 (2004), no. 1, 91-119, DOI 10.1215/S0012-7094-04-12514-X. MR2097358(2005i:46064)

[20] Terry A. Loring, Lifting solutions to perturbing problems in $C^{*}$-algebras, Fields Institute Monographs, vol. 8, American Mathematical Society, Providence, RI, 1997. MR.1420863 (98a:46090)

[21] Hiroki Matui and Yasuhiko Sato, $\mathcal{Z}$-stability of crossed products by strongly outer actions, Comm. Math. Phys. 314 (2012), no. 1, 193-228, DOI 10.1007/s00220-011-1392-9. MR2954514

[22] Hideki Nakamura, Aperiodic automorphisms of nuclear purely infinite simple $C^{*}$-algebras, Ergodic Theory Dynam. Systems 20 (2000), no. 6, 1749-1765, DOI 10.1017/S0143385700000973. MR,1804956(2002a:46089)

[23] Hiroyuki Osaka and N. Christopher Phillips, Crossed products of simple $C^{*}$-algebras with tracial rank one by actions with the tracial Rokhlin property, in preparation.

[24] N. Christopher Phillips, Equivariant K-theory and freeness of group actions on $C^{*}$ algebras, Lecture Notes in Mathematics, vol. 1274, Springer-Verlag, Berlin, 1987. MR.911880 (89k:46086)

[25] N. Christopher Phillips, Cancellation and stable rank for direct limits of recursive subhomogeneous algebras, Trans. Amer. Math. Soc. 359 (2007), no. 10, 4625-4652, DOI 10.1090/S00029947-07-03849-4. MR2320644(2009a:46134)

[26] N. Christopher Phillips, Crossed products by finite cyclic group actions with the tracial Rokhlin property, unpublished preprint (arXiv: math.OA/0306410).

[27] N. Christopher Phillips, The tracial Rokhlin property for actions of finite groups on $C^{*}$-algebras, Amer. J. Math. 133 (2011), no. 3, 581-636, DOI 10.1353/ajm.2011.0016. MR2808327(2012h:46116)

[28] N. Christopher Phillips, Every simple higher dimensional noncommutative torus is an AT algebra, preprint (arXiv: math.OA/0609783).

[29] Marc A. Rieffel, Applications of strong Morita equivalence to transformation group $C^{*}$ algebras, Operator algebras and applications, Part I (Kingston, Ont., 1980), Proc. Sympos. Pure Math., vol. 38, Amer. Math. Soc., Providence, R.I., 1982, pp. 299-310. MR679709 (84k:46046)

[30] Hiroshi Takai, On a duality for crossed products of $C^{*}$-algebras, J. Functional Analysis 19 (1975), 25-39. MR.0365160 (51 \#1413)

[31] Andrew S. Toms and Wilhelm Winter, Strongly self-absorbing $C^{*}$-algebras, Trans. Amer. Math. Soc. 359 (2007), no. 8, 3999-4029, DOI 10.1090/S0002-9947-07-04173-6. MR2302521 (2008c:46086)

Department of Mathematics, University of Oregon, Eugene, Oregon 97403-1222 